\title{
Efectos de la pedagogía y el compromiso educativo sobre el logro académico de los estudiantes de educación media
}

\author{
ASTORQUIZA BUSTOS, BILVER ADRIÁN \\ Facultad de Ciencias Contables, Económicas y \\ Administrativas \\ Universidad de Manizales (Colombia) \\ Correo electrónico:bilvera@umanizales.edu.co
}

\begin{abstract}
RESUMEN
Este artículo estudia por medio de los modelos lineales jerárquicos, la hipótesis de incidencia positiva que las variables de pedagogía y compromiso educativo generan sobre el desempeño académico. Para ello, son analizados las puntuaciones obtenidas por 62769 estudiantes de educación media en las pruebas PISA 2012 en 12 países. Al precisar que los procesos educativos adoptan una estructura jerárquica definida en dos niveles, estudiantes y planteles educativos, la especificación econométrica empleada conduce a resultados ostensiblemente mejores a los que se obtendría de una especificación tradicional. Entre los resultados se encuentra que el desempeño educativo en seis países es explicado en una mayor proporción por variables del entorno familiar y características del alumnado, mientras que en los seis restantes es explicado por el entorno escolar. De igual forma, los resultados validan la hipótesis de investigación formulada en el documento: la pedagogía y el compromiso educativo inciden positivamente sobre el desempeño académico. Así, la formulación de programas que fomenten dichas prácticas al interior de los planteles educativos debe formar parte de la agenda educativa. Por último, se encontró que el sexo del estudiante no aporta explicación alguna que refiera a existencia de brechas educativas pues los resultados son diferentes entre países, mientras que la no repetición de curso y la ausencia escolar, la dotación de elementos escolares en el hogar, el nivel de formación de los padres, entre otros factores, se asocian con sobresalientes resultados en el logro académico.
\end{abstract}

Palabras clave: análisis multinivel, entorno escolar, entorno familiar, logro académico, economía de la educación.

Clasificación JEL: C51; H52; I21; I28.

MSC2010: 62P20; 97B10. 


\title{
Effects of pedagogy and educational commitment on the academic performance of middle school students
}

\begin{abstract}
This article studies from the hierarchical linear models, the hypothesis of positive incidence that the variables of pedagogy and educational commitment generate on the academic performance. For this, the scores obtained by 62769 middle school students in the 2012 PISA tests in 12 countries are analyzed. By specifying that educational processes adopt a hierarchical structure defined in two levels: students and schools; the econometric specification used leads to results ostensibly better compared to those generated from a traditional specification. Among the results, we have that the educational performance in six countries is explained in a greater proportion by variables of the family environment and student's characteristics, while in six it is explained by the school environment. Similarly, the results confirm the research hypothesis formulated in the document: pedagogy and educational commitment have a positive impact on academic performance, therefore, the formulation of programs that encourage such practices within the schools should be part of the educational policy. Finally, it was found that the sex of the student does not provide any explanation that refers to the existence of educational gaps because the results are dissimilar between countries, while the non-repetition and absence from school, the provision of school elements at home, the level of parent training, among other factors, are associated with outstanding results in academic achievement.
\end{abstract}

Keywords: multilevel analysis, school environment, family environment, academic performance, economics of education.

JEL classification: C51; H52; I21; I28.

MSC2010: 62P20; 97B10.

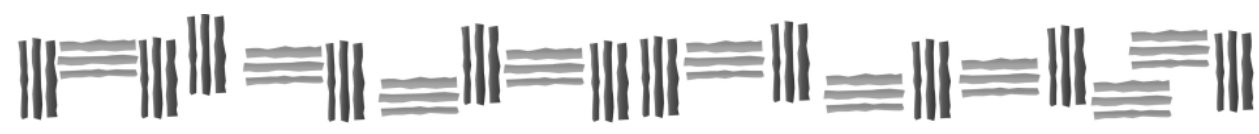




\section{Introducción.}

Los países con una población de elevadas cualificaciones académicas se identifican como aquellos que logran alcanzar un mejor bienestar social y desarrollo económico, por cuanto la educación es considerada como un instrumento indispensable para que los individuos potencien sus capacidades, sean competitivos y logren desenvolverse con mayor autonomía en cualquier situación de la vida cotidiana. Por esta razón, en la última década investigadores sociales en Colombia se interesaron en analizar los factores asociados con el incremento de las competencias de los ciudadanos, especialmente en el alumnado, trayendo nuevamente a discusión un tema que se ha tratado con rigurosidad desde la academia de los años sesenta por Schultz (1961), Denison (1962) y Becker (1962) en el campo de economía de la educación.

Sin embargo, pese a la existencia de numerosos estudios que han tratado este tema de coyuntura, y de las políticas definidas por el Ministerio de Educación Nacional de Colombia (ampliar la cobertura educativa, mejorar la eficiencia del sector educativo y la calidad de la educación), aún limita el entendimiento del por qué Colombia continúa ocupando los últimos lugares en pruebas internacionales que se especializan en medir las competencias de los alumnos en áreas claves para el desarrollo académico como la lectura, las matemáticas y las ciencias. Lo anterior, conduce a pensar en la existencia de especificidades que, posiblemente, no han sido abordadas.

De manera que, para explorar los componentes que conducen al bajo desempeño de los alumnos colombianos en pruebas internacionales como las diseñadas en el Programa Internacional para la Evaluación de Alumnos (PISA por sus siglas en ingles), esta investigación se orienta en responder la pregunta: ¿cuál es la incidencia entre las características del "entorno escolar" y del "entorno familiar" sobre el logro académico de los alumnos que presentaron las pruebas PISA 2012?, articulando de manera implícita dos efectos que van más allá de condensar características del clima escolar (número de bibliotecas, dotación escolar, jornadas educativas, entre otras) y que bien pueden ser intervenidas por el Estado en el corto y/o medio plazo a través de políticas públicas.

El primer efecto plantea una relación entre el alumno y el maestro, admitiendo que esta interacción propicia el aprendizaje y la transmisión de conocimientos. Lo anterior, junto con la percepción de intimidación escolar y la identificación de habilidades heterogéneas en los cursos se denomina en esta investigación como variables de "pedagogía". El segundo efecto es "compromiso educativo", el cual hace referencia a la identificación de los mecanismos implementados por los planteles educativos en pro de mejorar las condiciones académicas de sus alumnos, atribuido en cierta medida a la revisión continua de sus estructuras curriculares y su posterior comparación con otras entidades educativas tanto nacionales como internacionales. Por ello, pese a que la pregunta formulada busca contrastar la incidencia entre las características del entorno familiar y escolar sobre el logro académico, se precisa una mayor atención a estos dos efectos, pues forman parte del aporte que esta investigación realiza a la literatura de la Economía de la Educación.

Es así como al estudiar la incidencia de la pedagogía y el compromiso educativo sobre el logro académico, junto con los factores de entorno familiar y escolar es expuesta la relación disímil que estos componentes tienen sobre los sistemas educativos; en algunas economías los efectos se ven condicionados por el nivel de desarrollo. Por tal razón, se desarrolla un ejercicio comparativo entre los resultados de la modelación econométrica realizada para Colombia y un conjunto de países caracterizados por haber alcanzado en las pruebas PISA los primeros puestos (China-Shanghái, Corea, Finlandia y Singapur), posiciones intermedias (Alemania, Francia, Canadá, Bélgica) y posiciones bajas (Brasil, Chile, Uruguay) a partir de la técnica de modelos jerárquicos lineales, cuya relevancia teórica radica en reconocer la estructura jerarquía existente en el proceso educativo (alumnos y planteles educativos). 
La inferencia econométrica se realiza para cada uno de los países mencionados, pues tal como sugiere Hanushek (2008) se reconoce la existencia de brechas en ingresos nacionales, matrículas ofertadas, calidad de las instituciones educativas, años de educación promedio de la sociedad, entre otros factores que fácilmente pueden incidir en el desempeño de los alumnos. Sin embargo, considerarlos en la investigación se justifica en la medida que durante el análisis se exhiben algunas particularidades de sus sistemas educativos, dando paso a la definición de un perfil educativo meta. En consistencia con lo presentado, como hipótesis central se somete a prueba la afirmación: los efectos de pedagogía y compromiso educativo inciden positivamente en el logro académico, en especial, para aquellos alumnos que pertenecen a economías desarrolladas.

El desarrollo de esta investigación considera secuencialmente una breve apartado sobre enfoques metodológicos en economía de la educación (primera sección), una aproximación metodológica que sintetiza la fuente de los datos, la descripción de las variables explicativas y la especificación del modelo (segunda sección); el proceso de modelación, los resultados del ejercicio econométrico y la validación de los supuestos de la técnica (tercera sección); finalizando con las conclusiones y limitaciones del documento como apartado final.

\section{Revisión de Literatura.}

La economía de la educación distingue dos enfoques metodológicos que ostentan gran aceptación: el entorno familiar y el entorno escolar. El primer enfoque fue adoptado por autores como Coleman (1968), Spady (1973), Ermisch \& Francesconi (2001), Galor \& Zeira (1993), Lazear (1980), Mayer \& Peterson (1999), Vivas (2008), Jencks (1972), entre otros. Este enfoque analiza los efectos que tienen sobre el logro académico, el tamaño medio del hogar, la calidad de los entornos locales, la educación de los padres, los niveles de riqueza inicial con los que cuenta el hogar, bajo la premisa de que es en los hogares donde se fundamentan los valores y las herramientas iniciales que crean competencias y habilidades en los alumnos, del mismo modo que es en el hogar donde se toma la decisión de acceder o no al sistema educativo.

El segundo enfoque es desarrollado por Barrientos (2008), Betts y Shkolnik (2000), Casas, Gamboa y Piñeros (2002), Kane (2006), Raudenbush y Bryk (2002), Tobón, Posada y Ríos(2009). En este enfoque se argumenta que la calidad de los maestros, el tipo de plantel, las bibliotecas disponibles y el tamaño de la clase tienen efectos decisivos en el logro académico incluso superiores a los alcanzados por el entorno familiar, pues explican una mayor proporción de la varianza total del logro académico (Tobón et al., 2009, p. 326). A su vez, este enfoque exhibe la relevancia del entorno escolar en el proceso formativo del alumno en la medida en que, este espacio permite desarrollar sus capacidades cognitivas y, al estar inmerso en el ámbito académico, le brinda autonomía en la decisión de apropiarse o no del conocimiento impartido por los maestros durante las clases.

En consecuencia, al explorar los dos enfoques metodológicos presentados en esta investigación, se encontró una literatura dirigida más hacia las características asociadas al entorno escolar, en comparación con aquellos estudios que abordan el entorno familiar. Pese a ello, se hizo evidente que las argumentaciones expuestas en ambos casos condensan resultandos consistentes que merecen evaluarse en investigaciones que empleen el análisis jerárquico y, dado que, contribuyen en la fundamentación metodología de la estimación econométrica, se presentan a continuación una revisión de literatura nacional e internacional sobre este tema.

En Colombia, la investigación pionera en este campo fue realizada por Piñeros y Rodríguez (1998). Los autores emplearon datos del Instituto Colombiano para el Fomento de la Educación Superior (ICFES) de 1997 encontrando diferencias entre la calidad de la educación ofertada en instituciones del sector oficial y no oficial, sugiriendo que las instituciones oficiales cuentan con una desventaja en la calidad educativa. Adicionalmente, detallan que la diferencia de género está 
dada en favor de los niños y que los tiempos de desplazamiento hacia el centro educativo muestra una relación negativa con los resultados académicos.

Sin embargo, si bien se ha mantenido en vigencia el anterior postulado sobre calidad educativa a favor de las instituciones privadas y, por ende, contribuye en la explicación de la obtención de mejores resultados por parte de sus alumnos en pruebas nacionales como bien es documentado en la investigación de López (2010), autores como Núñez, Steiner, Cadena y Pardo (2002) encuentran que la eficiencia en la educación de los planteles públicos está dada para la población de los estratos socioeconómicos más bajos. De esta forma, al complementar la anterior apreciación con la investigación de Correa (2004) donde se explora el logro educativo con técnicas multinivel, es posible argumentar que las políticas educativas se justifican en la medida que conducen a mejorar la educación provista por las instituciones públicas, quienes terminaran afectando directamente la población que se beneficia de dicha educación, mención realizada por este último autor.

Sarmiento, Becerra y González (2000) y Gaviria y Barrientos (2001) prestan mayor atención a los efectos de los factores externos al entorno escolar sobre el logro académico. Los segundos autores manifiestan que los problemas en la educación son resultado de la estructura organizacional (distinción entre públicos y privados) y de los incentivos que tienen los alumnos al decidir si continúan con su proceso formativo. Entre tanto, los primeros afirman que el factor socioeconómico captado por la inversión en educación y el ingreso de los padres juega un papel sustancial en el aprendizaje, potencializado con el tipo de plantel educativo, en especial los privados.

Por su parte, Vivas (2008) establece que los factores asociados al entorno familiar y los efectos de vecindad que introducen las preferencias de localización espacial por parte de los agentes sociales, condicionan tanto el ingreso al sistema educativo como también los resultados alcanzados por los alumnos. Para este autor la educación permite el desarrollo de habilidades bajo el condicionante del nivel socioeconómico del alumno, el cual es un factor clave en el proceso de aprendizaje y contribuye en la explicación del máximo nivel de escolaridad que logran alcanzar. En este mismo orden de ideas, Ramos, Duque y Nieto (2012) también encuentra que la localización geográfica de los centros educativos justifica la brecha de los resultados obtenidos por el país en pruebas internacionales como las PISA en los años 2006 y 2009. Sin embargo, manifiesta que la desventaja se origina por efectos de entorno familiar como la educación de los padres, en especial el de las madres y, por tanto, mejorar la calidad de vida en los hogares debería constituirse como una política educativa.

La investigación de Zambrano (2013) ostenta una primera aproximación por introducir la pedagogía como estrategia educativa para el incremento en el desempeño educativo. El autor al indagar sobre las disparidades en la calidad de la educación a nivel alumno y plantel educativo para las pruebas de matemáticas en Colombia con datos del Estudio Internacional de Tendencias en Matemáticas y Ciencias (TIMSS por sus siglas en inglés), encontró que el 57.4\% de la varianza en el rendimiento es explicada por el nivel alumno, donde los mejores resultados provienen de los planteles educativos privados, que se encuentran emplazados en zonas geográficas urbanas, y determinado de igual forma por el gusto que sienten los alumnos por la materia y el plantel educativo. De esta forma, una de sus conclusiones se enfoca en invitar a las entidades educativas al fomento de procesos pedagógicos que refuercen estos gustos.

En el contexto internacional se destaca inicialmente el estudio realizado por Hanushek (1971) quien resalta la importancia del nivel socioeconómico del alumno y su efecto positivo sobre el logro académico, resultado atribuido a la utilización de un lenguaje más formal en el aula que permite una mejor comprensión de la enseñanza dictada por maestros con altas cualificaciones. En esta misma línea de ideas, autores como Galor y Zeira (1993) y Spady (1973) encontraron una relación directa entre desempeño educativo y nivel socioeconómico de los padres del alumno, el cual es determinado por el stock de capital humano que han acumulado. Por su 
parte, Lee y Barro (2001) argumentan que, si bien las características familiares tienen un efecto significativo sobre el desempeño de los alumnos, cursos de menor tamaño contribuye en mayor medida a mejorar los resultados educativos.

Entre tanto, Cohen, Raudenbush y Ball (2003) definen a los planteles educativos como facilitadores del aprendizaje, conjugando con anterioridad la premisa de Hanushek (2008), quien argumentó que la calidad de los maestros y el tamaño de la clase son elementos esenciales para el proceso formativo. Los autores encuentran que el tamaño de la clase es un factor a considerar pues si el alumno está rodeado de compañeros con una sana motivación y con un buen nivel educativo, es posible pensar que se fortalece y mejora el proceso formativo del aula de clases en conjunto; argumento que también es presentado por Hanushek, Markman y Rivkin (2003) al recomendar la interacción entre los alumnos con notas sobresalientes con los que presentan rezago formativo.

El potencial predictivo del análisis multinivel en los estudios que profundizan en la descomposición de la varianza no explicada del logro académico es consistente, puesto que, al comparar la investigación realizada por Orrego (2009) para España con los obtenidos en los estudios de Calero y Escardíbul (2007) y Martín, Asencio, Ordóñez y Romero (2009) se conjetura que las características propias del alumno priman sobre el entorno escolar (Cordero, Manchón, \& Simancas, 2012, p. 84), tal como se validan en los hallazgos de la presente investigación.

Finalmente, Cervini (2004) introduce el concepto de equidad en el aprendizaje para justificar la importancia de emprender análisis que conduzcan a mejorar el logro educativo. Para este autor todos los individuos deberían contar con un aprendizaje básico y cualquier desigualdad en el aprendizaje se configura como una violación al principio de igualdad. Partiendo de dicha precisión, el autor al analizar los resultados en las pruebas de matemáticas y lenguaje de 126,590 alumnos argentinos encontró que factores como repetir cursos, la restricción en el acceso al capital cultural como los libros, y las condiciones familiares como el estado laboral de los padres que implique la vinculación del alumno al mercado laboral, están asociados con bajos rendimientos.

\section{Aproximación Metodológica.}

\subsection{Descripción de datos y variables explicativas.}

Los datos de la investigación provienen de PISA 2012, los cuales permiten evaluar la calidad de la educación de los países miembros y asociados, como en el caso de Colombia ${ }^{1}$, de la Organización para la Cooperación y el Desarrollo Económico (OCDE). PISA evalúa las competencias en lectura, matemáticas y ciencias de los alumnos de grado séptimo hasta once de bachiller con edades entre los 15 y los 16 años con el objetivo de revisar los conocimientos, aptitudes y competencias adquiridas durante el proceso formativo del alumno.

Para conocer los efectos que las variables del entorno escolar, del alumno y, puntualmente, el de pedagogía y compromiso educativo tienen sobre el logro académico se plantea un análisis comparativo entre los resultados alcanzados por los alumnos colombianos y los alumnos de algunas economías que se han ubicado en las primeras posiciones de las pruebas (China-Shanghái, Corea, Finlandia, Singapur), en posiciones intermedias (Alemania, Francia, Canadá, Bélgica) y en posiciones bajas (Brasil, Chile, Uruguay). De lo anterior, se consolida una muestra total de 62 769 observaciones, las cuales representan cerca de 3637408 alumnos una vez se introduce el factor de expansión.

\footnotetext{
${ }^{1}$ Este país ingreso como miembro de la organización en el año 2018.
} 
En lo referente a la elección de variables, se sustentan en la definición de los modelos de oferta y demanda de servicios educativos realizada por Piñeros \& Rodríguez (1998) y en el lineamiento seguido por Cano (2006) al introducir variables de percepción, tanto del maestro como de los directores del plantel educativo y de reconocimiento autónomo del alumno. No obstante, el sustento conceptual emplea parte del argumento teórico de Vivas (2008), donde se fundamenta la oferta como la asociación entre los resultados y las dotaciones escolares que el alumno posee dentro de su proceso formativo; y la demanda como los factores que permiten acceder a la educación.

Inicialmente, la variable explicativa definida como compromiso educativo se construye a partir de la respuesta sí o no a la pregunta ¿el plantel educativo usa las evaluaciones de los alumnos para (...)? Los tres componentes principales de la variable son: i) autoevaluación y mejoramiento de la estructura curricular, ii) comparación con otras instituciones y iii) eficiencia docente en la transmisión del conocimiento. Por tanto, los planteles que afirman realizar al menos uno de los componentes se asocia con el compromiso educativo por cuanto evaluar la calidad de la educación demuestra el interés que tienen por mejorar continuamente, mientras que los planteles que no realizan ninguna de estas actividades se considera que no están comprometidos con mejorar la educación.

Ahora, para explorar los aspectos pedagógicos fue necesario recurrir al análisis de la percepción del clima escolar contestadas por el rector al siguiente interrogante: ¿en su plantel, en qué medida es el aprendizaje de los alumnos impedido por los siguientes fenómenos?, seleccionando cuatro variables: i) intimidación escolar, ii) baja expectativa que los alumnos tienen hacia los maestros, iii) la consideración de si los maestros son estrictos y iv) si existen habilidades heterogéneas entre los alumnos de un mismo curso. Esta última variable profundiza en el hecho en que los maestros enseñan a los alumnos con niveles cognitivos disimiles dentro de la misma clase.

En complementariedad con las variables explicativas que se introducen para mejorar la especificación econométrica y que exhiben características del alumno y de su entorno familiar permiten indagar las posibles diferencias en los resultados originadas por cuestiones de sexo; el nivel de escolaridad y la vinculación laboral de los padres (ocupados o desempleados); el tipo de hogar donde se examina si el alumno vive con el padre y la madre, o por el contrario, convive con uno de ellos u otros familiares; y la dotación en el hogar que evalúa la tenencia de al menos uno de los siguientes recursos: poseer escritorio, computador, conexión a internet, literatura clásica, DVD o un lugar de estudio.

En cuanto a las características del entorno escolar se tiene el tipo de plantel (instituciones públicas y privadas), la localización del plantel, el tamaño de la clase, la diferencia de resultados originadas en los planteles con maestros de tiempo parcial frente a aquellos que no y el ofrecimiento de actividades estudiantiles como banda, periódico estudiantil, equipos deportivos y clubes de matemática, ajedrez, informática o arte. Esta última variable se articula bajo la premisa de que actividades extracurriculares pueden estar asociadas con buenos resultados por cuanto permite la interacción entre alumnos y la puesta en práctica de ciertos conocimientos.

Así mismo, se introducen variables características de la demanda educativa desde la dimensión de antecedentes escolares: repetición de curso y absentismo. En la investigación de (Hernández, Rosário, de Tejada, Martínez, \& Ruiz, 2006, p. 418) se expone que la primera variable es de comprobada relevancia en la explicación del "éxito escolar”, y si bien es probable que se encuentre condicionada por factores que no dependen exclusivamente del alumno, su inclusión obedece a una robusta especificación del modelo. Justamente, se espera que el resultado obtenido por los alumnos que repiten al menos una vez un curso del bachillerato y aquellos que se ausentan en la misma frecuencia, obtengan menores resultados en comparación con los que nunca han repetido curso y tampoco se han ausentado. 
De igual forma, se estudia si el plantel educativo presenta escasez de maestros cualificados en las áreas evaluadas en PISA, de materiales de instrucción (libros), edificios escolares y aulas de clase, desde la visión de la capacidad o no que el plantel tenga para solucionar la carencia de estos factores. El signo esperado, la metodología de codificación y las estadísticas descriptivas están en los anexos 1 y 2 respectivamente.

Finalmente, la variable dependiente corresponde a la media muestral de los valores plausibles de PISA 2012 como proxy del logro académico. En la prueba se evalúan las competencias en lectura, ciencias y matemáticas, donde cada competencia cuenta con cinco valores plausibles, lo cual hace necesario calcular el valor promedio, dando como resultado un único valor que no se ve afectado por la agrupación debido a que las puntuaciones siguen una distribución normal, siendo uno de los supuestos fundamentales en los modelos multinivel, el cual es probado más adelante. La forma funcional de las variables junto con los principales estadígrafos se presenta en los anexos 1 y 2 respectivamente.

\subsection{Síntesis general de los modelos lineales jerárquicos.}

La fundamentación analítica de los modelos jerárquicos se expone con detenimiento en los trabajos de Bryk y Raudenbush (1992), Goldstein (1995), Hox (2002) y Snijders y Bosker (1999). No obstante, esta investigación sigue la notación y línea argumental de Gaviria y Castro (2005), precisando que el desarrollo de estos modelos es propio del campo de la educación y dada la facilidad analítica de los mismos, los investigadores económicos adoptaron la teoría y la complementaron con técnicas y formulaciones econométricas de mayor robustez. El desarrollo estadístico del modelo jerárquico y del modelo no condicionado se presenta a continuación.

$$
\gamma_{i j}=\beta_{0 j}+\beta_{1 j} X_{1 i j}+\varepsilon_{i j}
$$

La ecuación 1 representa la especificación general de un modelo multinivel, donde los subíndices i y j indican el alumno y el plantel educativo, por tanto, las variables descritas con ellos hacen alusión al comportamiento del modelo así: $X_{1 j}$ es la variable asociada a las características del alumno $i$ que pertenece al plantel $j, \gamma_{i j}$ es el indicador del logro académico promedio del alumno, $\beta_{0 j}$ es el rendimiento esperado del plantel cuando $X_{1 j}$ es igual a cero y $\beta_{1 j}$ capta el incremento (disminución) en el logro académico causado por la variación unitaria de las características del alumno. Entre tanto, $\varepsilon_{i j}$ es el término de la ecuación que se aparta del rendimiento esperado del alumno según su vector de características, asumiendo que la varianza es homogénea en todos los planteles $\varepsilon_{i j} \sim N\left(0, \sigma_{\varepsilon}^{2}\right)$. Como $\beta_{0 j}$ y $\beta_{1 j}$ son los valores de cada plantel, al contar el modelo con una distribución bivariada normal en el sentido en que cada coeficiente integra un componente invariable y uno aleatorio dada la heterogeneidad entre planteles, los coeficientes se convierten en variables aleatorias en el nivel macro, lo cual hace que sean representadas así:

$$
\begin{aligned}
& \beta_{0 j}=\beta_{0}+\mu_{0 j} \\
& \beta_{1 j}=\beta_{1}+\mu_{1 j}
\end{aligned}
$$

Una vez se descompone $\beta_{0 j}$ (ecuación 2) se obtiene una parte invariable captada por $\beta_{0}$ (logro académico promedio en la población de planteles) y una parte aleatoria $\mu_{0 j}$ (logro 
diferencial del plantel $j$ ) que forma parte del modelo en el segundo nivel. De modo análogo, $\beta_{1 j}$ (ecuación 3) se descompone en $\beta_{1}$ que conforma la parte invariante y representa la variación media del rendimiento en la prueba PISA causada por la variación de $X_{1}$ en la población de planteles, y un término $\mu_{1 j}$ que hace parte de la aleatoriedad del modelo en el segundo nivel y representa el incremento diferencial en el rendimiento por el aumento de $X_{1}$ en plantel $j$.

$$
\begin{array}{ll}
E\left(\beta_{0 j}\right)=\beta_{0} & \mathrm{~V}\left(\beta_{0 j}\right)=\sigma_{\mu_{0}}^{2} \\
E\left(\beta_{1 j}\right)=\beta_{1} & \mathrm{Cov}\left(\beta_{0 j}, \beta_{1 j}\right)=\sigma_{\mu_{0}}^{2}
\end{array}
$$

La ecuación 4 muestra las propiedades estadísticas de la distribución bivariada de $\beta_{0 j} \mathrm{y}$ $\beta_{1 j}$. El supuesto de la variación en los planteles o macro-niveles es fundamental cuando se aborda el análisis de modelos jerárquicos, si esta variación no existiera, este tipo de modelación no sería adecuada. Así, al introducir variables del segundo nivel, el modelo referente al micro-nivel no sufre ningún cambio (ecuación 1), mientras que el macro-nivel (ecuaciones 2 y 3) sufre alteraciones quedando de la siguiente forma:

$$
\begin{gathered}
\beta_{0 j}=\beta_{00}+\beta_{01} Z_{1 j}+\mu_{0 j} \\
\beta_{1 j}=\beta_{10}+\beta_{11} Z_{1 j}+\mu_{1 j}
\end{gathered}
$$

Al combinar las anteriores ecuaciones se llega a una notación compacta de los modelos multinivel bietápico para $\mathrm{N}$ variables explicativas asociadas a los $i$ alumnos (nivel 1) y $\mathrm{L}$ variables explicativas asociadas a los planteles (nivel 2) de la siguiente forma:

$$
\begin{aligned}
& \gamma_{i j}=\beta_{0 j}+\sum_{n=1}^{N} \beta_{n j} X_{n i j}+\varepsilon_{i j} \text { donde } \varepsilon_{i j} \sim N\left(0, \sigma_{\varepsilon}^{2}\right) \\
& \beta_{n j}=\beta_{n 0}+\sum_{l=1}^{L} \beta_{n j} Z_{l j}+\mu_{n j} \text { donde } \mu_{n j} \sim N(0, T)
\end{aligned}
$$

El modelo no condicionado es una herramienta metodológica que permite fundamentar la implementación de las jerarquías; al no contar con variables explicativas indica si realmente es adecuado incluir variables en los niveles definidos a partir de la regresión. Si al estimarse este modelo se obtiene como resultado una varianza estadísticamente significativa e igual a cero para los macro-niveles, no tendría sentido incluir variables explicativas. De las ecuaciones 9 y 10 se define que $\sigma_{\mu 0}^{2}$ es la varianza entre los planteles educativos y $\sigma_{\varepsilon}^{2}$ es la varianza entre los alumnos.

$$
\begin{array}{rc}
\gamma_{i j}=\beta_{0 j}+\varepsilon_{i j} & \text { Nivel uno } \\
\beta_{0 j}=\beta_{0}+\mu_{0 j} & \text { Nivel dos } \\
\gamma_{i j}=\beta_{0}+\mu_{0 j}+\varepsilon_{i j} \text { donde } & \varepsilon_{i j} \sim N\left(0, \sigma_{\varepsilon}^{2}\right) \text { y } \mu_{0 j} \sim N\left(0, \sigma_{\mu 0}^{2}\right)
\end{array}
$$

\section{Resultados de la modelación econométrica.}

La estimación de los componentes del modelo no condicionado son el punto de partida del análisis. En la tabla 1 se muestran los coeficientes estimados para $\beta_{0 \mathrm{ij}}$ (parte fija del modelo), los 
cuales representan el intercepto del modelo no condicionado, es decir, el logro académico promedio de los alumnos en las pruebas PISA 2012 junto con sus respectivos errores típicos (entre paréntesis); se confirma la significancia estadística de los estimadores a partir del test de Wald. Entre tanto, los coeficientes de la parte aleatoria también son significativos y dado que corresponden a las varianzas estimadas para cada nivel, se encuentra una variación sin explicar del logro académico promedio de los alumnos $\left(\sigma_{e}^{2}\right)$ y los planteles $\left(\sigma_{\mu 0}^{2}\right)$, haciendo que la estrategia empírica busque disminuir dicha varianza una vez se introducen de manera secuencial las variables explicativas.

Tabla 1. Estimación del modelo no condicionado por países.

\begin{tabular}{|l|ccccc|}
\hline \multirow{2}{*}{ Países } & Parte Fija & \multicolumn{2}{c}{ Parte Aleatoria } & $\begin{array}{c}\text { Correlación } \\
\text { intraclase }\end{array}$ & IGLS Deviance \\
\cline { 2 - 6 } & $\boldsymbol{\beta}_{\mathrm{Oij}}$ & $\sigma^{2}$ & $\sigma^{2}$ & $\boldsymbol{O}_{e}$ & -2*loglikelihood \\
\hline Alemania & $518.102(5.150)$ & $4834.029(436.093)$ & $3099.997(89.157)$ & $\mathbf{3 9 . 0 7 \%}$ & 29337.834 \\
Bélgica & $521.542(4.572)$ & $5102.911(435.671)$ & $3805.230(105.065)$ & $\mathbf{4 2 . 7 2 \%}$ & 63688.148 \\
Brasil & $418.984(4.302)$ & $2864.036(355.146)$ & $2792.954(84.738)$ & $\mathbf{4 9 . 3 7 \%}$ & 34039.971 \\
Canadá & $517.956(1.419)$ & $1360.661(93.442)$ & $5125.435(66.296)$ & $\mathbf{7 9 . 0 2 \%}$ & 194049.373 \\
Chile & $452.153(4.392)$ & $3521.707(266.293)$ & $2612.916(63.551)$ & $\mathbf{4 2 . 5 9 \%}$ & 46839.722 \\
Colombia & $401.623(2.686)$ & $1988.986(232.577)$ & $2805.374(56.842)$ & $\mathbf{5 8 . 5 1 \%}$ & 59513.127 \\
Corea & $548.187(4.632)$ & $2960.452(377.183)$ & $4664.718(166.914)$ & $\mathbf{6 1 . 1 8 \%}$ & 44455.213 \\
Finlandia & $517.782(1.870)$ & $680.910(105.455)$ & $6072.539(119.203)$ & $\mathbf{8 9 . 9 2 \%}$ & 73614.412 \\
Francia & $503.662(5.186)$ & $5013.923(536.423)$ & $3366.693(95.325)$ & $\mathbf{4 0 . 1 7 \%}$ & 30108.111 \\
Shanghái & $600.848(5.276)$ & $3934.754(399.032)$ & $3587.886(150.620)$ & $\mathbf{4 7 . 6 9 \%}$ & 46969.550 \\
Singapur & $560.369(4.863)$ & $3392.014(393.096)$ & $5849.954(188.206)$ & $\mathbf{6 3 . 3 0 \%}$ & 51516.848 \\
\hline \multirow{2}{*}{ Uruguay } & $415.469(4.631)$ & $3354.039(355.446)$ & $3392.987(97.939)$ & $\mathbf{5 0 . 2 8 \%}$ & 34003.374 \\
\hline
\end{tabular}

Fuente: Elaboración propia a partir de PISA 2012 (OCDE, 2014).

Ahora bien, con el fin de cuantificar cuál de los efectos asociados al nivel alumno o plantel aporta mayor explicación a la varianza desconocida del modelo, se procede a la estimación del coeficiente de correlación intraclase, estadístico que permite generar una primera aproximación sobre la dependencia del logro académico, ya sea por cuestiones asociadas al entorno familiar y del alumno, o del entorno escolar. De esta forma, al calcular el coeficiente con la varianza del nivel alumno se encuentra que en Finlandia (89.92\%), Canadá (79.02\%) Singapur (63.3\%), Corea (61.18\%), Colombia (58.51\%) y Uruguay (50.28\%) el total de la varianza desconocida es explicada, en mayor proporción, por la heterogeneidad que presentan las características del alumno y de su entorno familiar. Mientras en Alemania (39.07\%), Francia (40.17\%), Bélgica (42.72\%), Chile (42.59\%), Shanghái (47.69\%), Brasil (49.37\%) la diferenciación del logro académico es explicada por el nivel plantel. Ante estos resultados, la investigación se encauza en introducir variables que permitan disminuir la varianza no explicada, todo lo anterior, introduciendo el factor de expansión como ponderador de la varianza. En la tabla 2 se presentan los coeficientes estimados para cada uno de los países.

Los resultados sugieren que el compromiso educativo incide positivamente en el logro académico, pero no es un efecto generalizado en todas las economías en estudio. La variable 
presentó el signo esperado y una significancia estadística en Finlandia (15.540), Bélgica (24.620), Singapur (15.640) y Shanghái (16.783), países que se caracterizan por mantenerse entre las primeras posiciones de PISA. Por tanto, configurar y alentar el desarrollo de estas prácticas al interior de los planteles en Colombia posiblemente generarían una mejora en el desempeño de los alumnos pues, claramente, los perfiles meta en educación deben estar orientados a implementar estrategias que mejoren sus cualificaciones y éstas deberían estar sustentadas en los procesos exitosos seguidos por otros países.

La percepción de intimidación escolar en los planteles educativos es un fenómeno de varias décadas, sin embargo, recientemente ha cobrado una mayor atención por cuanto ha logrado permear el equilibrio psicológico y la forma en cómo se relacionan los alumnos dentro de distintos contextos sociales. El modelo estimado muestra como en Bélgica, Canadá, Finlandia, Francia, Shanghái y Singapur la presencia de intimidación escolar impide el aprendizaje en los alumnos y está correlacionada con la obtención de 12.56 y 29.97 puntos menos en el logro académico promedio.

Las bajas expectativas que el alumno forme sobre su maestro impiden el aprendizaje por cuanto el intercambio de conocimiento puede verse afectado por la falta de credibilidad en los temas enseñados. Es así como en Corea, Singapur, Alemania, Colombia, Bélgica, Canadá, Chile y Uruguay la presunción es comprobada, a manera de hipótesis, por el modelo. Los resultados dan paso a repensar en la importancia que tiene la relación entre el alumno y el maestro, pues al ser el canal directo de transmisión del conocimiento es clave que no sólo se transmita con claridad, sino que también se mantenga al alumno interesado en el aprendizaje.

La presencia de maestros estrictos en los planteles está asociada con mayores logros académicos en Alemania, Canadá y Francia, arrojando un signo contrario al sugerido para su interpretación; lo anterior expone dos claros retos teóricos producto de la falta de definición técnica que las pruebas PISA auscultan de la variable. La primera de ellas es que la connotación de severidad de un maestro puede estar asociada con la rigurosidad con que se transmite el conocimiento; mientras que la segunda se logra orientar en la formalidad del lenguaje al interior de los cursos; estando ambas interiorizadas en la investigación.

Ahora bien, cursos con habilidades heterogéneas entre alumnos se asocian con resultados menores en Corea, y Francia, mientras que Brasil exhibe un resultado contrario al sugerir que los planteles con cursos caracterizados por contar con niveles de habilidades heterogéneas obtienen 13.534 puntos más que los cursos con cierta homogeneidad. Si bien, Brasil no capta el sentido que se desprende teóricamente de la variable expuesta, y pese a la falta de significancia de gran parte de los países, es adecuado sugerir que la política educativa debería fomentar la estabilización de las habilidades cognitivas del alumno al interior de los cursos, o en su defecto, propiciar espacios adicionales donde los alumnos puedan nivelarse, todo ello a partir de la incorporación de herramientas de acompañamiento escolar como sugiere Ghouali (2007). 
Tabla 2. Modelo entorno familiar, escolar, factores pedagógicos y de compromiso educativo

\begin{tabular}{|c|c|c|c|c|c|c|c|c|c|c|c|c|}
\hline \multirow[b]{2}{*}{ Variables Paises } & \multicolumn{4}{|c|}{ PUNTA_ES ALTOS EN PRUJPRSPISA 2012} & \multirow{2}{*}{$\begin{array}{c}\text { RFGPEICIA } \\
\text { Colombia }\end{array}$} & \multicolumn{4}{|c|}{ PUNTAJES INTEFMIEXOS EN PRIEBAS PISA 2012} & \multicolumn{3}{|c|}{ PWNTAJES BRJOS ENPRIEBRSPISR 2012} \\
\hline & Corea & Finlandia & Shanghái & Singapur & & Francia & Alemania & Bélgica & Canadá & Chile & Brasil & Uruguay \\
\hline Miño & $-2.203(2.956)$ & $-15.026(2034)^{\star}$ & $4.322(1.935)^{\text {\#" }}$ & $-9.014(2.479)^{\star}$ & $14.691(1.488)^{\star}$ & $7.996(2.344)^{*}$ & $8.351(2.215)^{*}$ & $8.175(1.81\})^{\star}$ & $-2.865(1.195)^{\text {\#" }}$ & $15.415(1.618)^{*}$ & $10.734(2.278)^{\star}$ & $7.859(2.285)^{\star}$ \\
\hline Plantel Privado & 4.116(8.937) & $1.935(7.207)$ & $31.759(13.537)^{\text {th }}$ & $-37.097(28.597)$ & $19.626(6.968)^{*}$ & $9.040(8.627)$ & $16.874(17.239)$ & $47.181(7.722)^{*}$ & $35.270(4.106)^{\star}$ & $31.992(6.505)^{*}$ & $67.284(10.173)^{*}$ & $46.798(8.386)^{*}$ \\
\hline Plartel Lo alizado en Ciudad & $22.724(11.737)^{\text {th }}$ & $-4.003(3.354)$ & $0.000(0.000)$ & $0.000(0.000)$ & $18.828(4.697)^{*}$ & $-7.753(7.716)$ & $-6.800(10.127)$ & $-7.762(9.074)$ & $-0.217(2.380)$ & $19.707(6.042)^{*}$ & $5.949(5.952)$ & $17.923(5.412)^{*}$ \\
\hline Repiterte & $-20.193(6.137)^{*}$ & $-69.891(8.756)^{\star}$ & $-41.246(8.967)^{\star}$ & $-61.161(8.694)^{\star}$ & $-35.649(1.484)^{*}$ & $-22.415(5.387)^{*}$ & $-27.899(3.522\}^{\star}$ & $-56.022(2.856)^{\star}$ & $-65.171(3.111)^{\star}$ & $-53.298(4.087)^{\star}$ & $-39.130(3.073)^{\star}$ & $-54.260(3.483)^{*}$ \\
\hline Ausentarse & $-74.223(9.152)^{*}$ & $-41.461(3.085)^{\star}$ & $-49.357(15.738)^{*}$ & $-26.673(3.472)^{\star}$ & $-7.225(4.036)^{\mathrm{H}}$ & $-17.316(3.896)^{*}$ & $-11.569(5.728)^{*+}$ & $-27.294(4.862)^{\star}$ & $-24.116(1.401)^{*}$ & $-15.482(3.402\}^{\star}$ & $-2.386(2.512)$ & $-4.129(2.355)^{*+}$ \\
\hline Macke Eouxación Técnicá Universitaria & $9.093(2.592)^{\star}$ & $20.377(2.475)^{\star}$ & $-2.591(2.671)$ & $7.100(2.988)^{\mathrm{H}}$ & $8.587(1.669)^{*}$ & $9.407(2.698)^{*}$ & $4.152(2.456)^{n+\text { tot }}$ & $1.078(2.436)$ & $9.214(1.359)^{\star}$ & $7.899(1.946)^{\star}$ & 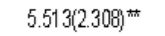 & $7.730(2.760)^{\star}$ \\
\hline Macre Empleada & $19.767(8.492)^{\text {H }}$ & $25.605(4.592)^{\star}$ & $17.908(7.797)^{\text {th }}$ & $19.885(7.600)^{*}$ & $11.144(2.408)^{*}$ & $20.314(5.380)^{*}$ & $-8.832(5.788)$ & $9.625(4.352)^{* *}$ & $18.489(2.915)^{\star}$ & $5.711(3.367)^{* *+}$ & $9.735(3.273)^{\star}$ & $0.424(4.824)$ \\
\hline Pacre Educación Técnical Universitaria & $9.746(2.588)^{*}$ & $13.701(2.419)^{\star}$ & $10.266(2.461)^{*}$ & $12.822(3.559)^{*}$ & $4.300(1.677)^{*}$ & $9.766(2.839)^{*}$ & $5.440(2.573)^{\text {\#t }}$ & $3.717(2.584)$ & $9.129(1.395)^{*}$ & 4. $191(1.928)^{3 \mathrm{~K}}$ & $7.193(2.225)^{*}$ & $4.299(2.627)^{\mathrm{mat}}$ \\
\hline Padre Empleado & $-6.668(8.646)$ & $8.850(4.284)^{\mathrm{Ho}}$ & $-9.488(6.560)$ & $0.640(8.815)$ & $0.432(3.180)$ & $1.531(6.763)$ & $5.917(7.821)$ & $15.042(5.224)^{*}$ & $2.645(3.738)$ & $-10.467(4.867)^{\text {to }}$ & $7.779(4.7 \mathrm{dd})^{\mathrm{nt \textrm {N }}}$ & $6.536(5.277)$ \\
\hline Vive Con Padre Y Macte & $18.109(3.866)^{\star}$ & $16.406(2.345)^{*}$ & $0.820(2.358)$ & $14.416(3.489)^{*}$ & $14.433(1.576)^{*}$ & $5.971(2.938)^{\text {\#t }}$ & $1.870(3.107)$ & $8.709(2.652)^{\star}$ & $11.852(1.605)^{\star}$ & $4.856(1.910)^{\text {\#t }}$ & $6.970(2.387)^{\star}$ & $14.787(2.635)^{\star}$ \\
\hline Dotación Hogar & $1.147(2.253)$ & $28.296(1.914)^{\star}$ & $7.230(1.869)^{\star}$ & $13.479(2.905)^{*}$ & $6.081(2.021)^{\star}$ & $27.414(2.450)^{*}$ & $10.986(2.379)^{*}$ & $15.859(1.787)^{*}$ & $17.760(1.192)^{\star}$ & $11.566(1.788)^{*}$ & 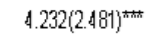 & $6.015(2.17 d)^{\star}$ \\
\hline Tamaño Clase & $-9.942(17.404)$ & 53.467(29.238) & $11.345(9.248)$ & $-20.922(17.699)$ & $14.828(8.343)^{*}$ & $60.015(8.333)^{*}$ & $49.839(8.666)^{\star}$ & $15.249(10.726)$ & $20.445(2.324)^{\star}$ & $-1.736(8.924)$ & $29.314(11.575)^{*+*}$ & $13.440(5.391)^{\mathrm{H}}$ \\
\hline Mæestros Tiempo Parcial & $7.757(10.606)$ & $-0.304(5.284)$ & $-27.527(10.676)^{\mathrm{H}}$ & $-4.487(14.052)$ & $1.340(4.729)$ & $51.043(51.627)$ & $0.000(0.000)$ & $81.758(16.978)^{\star}$ & $13.575(4.640)^{\star}$ & 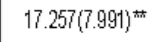 & $-1.211(8.350)$ & $25.998(14.612)^{\text {H }}$ \\
\hline Habilidades. Heterogéneas & $-25.956(12.552)^{\text {;* }}$ & $-9.842(6.755)$ & $21.946(16.553)$ & $-3.529(18.160)$ & $-11.021(8.154)$ & $-27.183(12.228)^{*+*}$ & $5.836(15.072)$ & $-21.414(13.855)$ & $-3.334(3.702)$ & $-6.283(8.873)$ & $13.534(7.085)^{\text {tot }}$ & $-5.164(10.271)$ \\
\hline EscasezMateriales & $-0.743(8.266)$ & $-1.766(3.54 d)$ & $10.553(10.301)$ & $13.507(11.344)$ & $-14.232(7.437)^{\text {mat }}$ & $-35.134(7.113)^{\star}$ & $12.036(9.664)$ & $-13.831(7.751)^{\text {H+ }}$ & $-2.123(2.481)$ & $-5.335(6.150)$ & $-9.385(6.007)$ & $-0.751(5.114)$ \\
\hline Escasez de Maestros & $7.538(9.282)$ & $-2.121(3.060)$ & $-10.409(8.051)$ & $-3.749(8.552)$ & $0.846(3.619)$ & $1.820(6.374)$ & $-24.488(8.656)^{\star}$ & $-10.556(6.977)$ & $-0.815(2.237)$ & $-3.423(4.478)$ & $-6.324(5.286)$ & $0.986(4.740)$ \\
\hline Escasez Espacios Educativos & $25.957(12.691)^{\text {th }}$ & $5.182(5.165)$ & $13.010(11.353)$ & $5.639(8.300)$ & $-1.123(6.705)$ & $21.786(10.668)^{*}$ & $2.142(11.188)$ & $4.864(10.436)$ & $7.159(2.747)^{*}$ & $-9.283(7.215)$ & $-6.298(9.656)$ & $-8.998(6.998)$ \\
\hline Escasez de Edificios & $-11.587((12.140)$ & $-3.826(5.061)$ & $-11.013(13.058)$ & $3.688(7.996)$ & $0.835(8.657)$ & $5.017(9.889)$ & $-10.036(11.373)$ & $-11.558(10.436)$ & $-0.853(2.844)$ & $-7.753(7.036)$ & $8.168(10.405)$ & $-15.794(6.160)$ \\
\hline Actividaces Escolares & $13.565(8.197)^{\text {㷊 }}$ & $0.000(0.000)$ & $55.212(9.615)^{\star}$ & $70.680(17.254)^{\star}$ & $-6.571(8.465)$ & $44.933(28.497)$ & $47.973(19.044)^{*+1}$ & $-18.550(21.515)$ & $17.185(3.994)^{\star}$ & $33.851(13.146)^{*}$ & $-42.820(11.684)^{*}$ & $-110.506(10.821)^{*}$ \\
\hline Compromiso Educativo & $10.378(8.094)$ & $15.540(5.804)^{\star}$ & $16.783(8.218)^{\text {to }}$ & $15.640(8.656)^{\text {tot }}$ & $5.261(4.178)$ & $-2.496(13.455)$ & $11.766(16.129)$ & $24.620(13.459)^{\text {tw }}$ & $-1.155(2.807)$ & $1.445(6.621)$ & $5.838(5.816)$ & $-4.132(9.279)$ \\
\hline Intimidación Escolar & $-9.039(11.913)$ & $-13.322(7.902)^{7+m}$ & $-19.240(9.805)^{\text {toto }}$ & $-28.234(9.797)^{\star}$ & $-5.631(5.767)$ & $-29.967(6.795)^{\star}$ & $-18.045(18.287)$ & $-26.997(8.561)^{\star}$ & $-12.564(3.486)^{*}$ & $6.604(7.463)$ & $0.747(7.090)$ & $-0.348(5.756)$ \\
\hline Bajas Expectativas Del Alumno & $-29.142(13.456)^{*}$ & $-0.968(3.069)$ & $-4.124(11.057)$ & $-21.235(9.052)^{\text {Ht }}$ & $-8.868(5.253)^{\star}$ & $-6.534(7.953)$ & $-22.757(9.813)^{\text {to }}$ & $-17.999(7.800)^{\text {\#t }}$ & $-7.771(2.542)^{\star}$ & $-29.828(7.246)^{\star}$ & $-1.644(8.680)$ & $-20.942(7.233)^{*}$ \\
\hline Maestros Estrictos & $12.336(9.839)$ & $-2.870(3.511)$ & $4.339(11.776)$ & $11.597(9.068)$ & $0.270(6.113)$ & $24.297(7.478)^{\star}$ & $16.690(10.255)^{\star}$ & $12.784(8.014)$ & 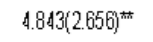 & $10.379(7.682)$ & $3.318(7.553)$ & $6.598(6.210)$ \\
\hline interc exto & $511.587(28.127)^{*}$ & $474.629(13.008)^{*}$ & $553.582(18.158)^{*}$ & $575.873(27.403)^{\star}$ & $374.319(13.803)^{\star}$ & $388.542(55.432)^{*}$ & $515.815(24.366)^{\star}$ & $434.068(24.837)^{\star}$ & $461.697(7.977)^{\star}$ & $422.887(15.669)^{\star}$ & $339.951(18.799)^{\star}$ & $391.627(18.947)^{\star}$ \\
\hline Observaciones & 3902 & 6344 & 4215 & 4437 & 5451 & 2694 & 2644 & 5671 & 16923 & 4316 & 3115 & 3057 \\
\hline Escuelas & 147 & 282 & 146 & 152 & 306 & 200 & 196 & 257 & 839 & 194 & 166 & 169 \\
\hline$\left(\sigma_{t}^{2}\right)$ & $4486.256(152.135)$ & $5260.631(95.467)$ & $3494.499(143.002)$ & $5559.374(168.952)$ & $2464.900(53.271)$ & $3109.079 .(82.594)$ & $2975.655(85.028)$ & $3423.542(94.257)$ & $4760.667(60.516)$ & $2425.257(56.992)$ & $2580.865(78.729)$ & $3054.365(81.425)$ \\
\hline$\left(\sigma_{\mu, H}^{a}\right)$ & 1981.168(262.070) & $347.846(49.930)$ & $2154.772(300.349)$ & $2042.664(265.330)$ & $1052.518(112.405)$ & $1554.427(185.305)$ & $3037.632(336.886)$ & $2613.804(269.020)$ & $732.043(47.838)$ & $1387.187(140.483)$ & $1114.047(148.685)$ & $787.660(119.414)$ \\
\hline Modelo Nulo & 7625.17 & 6753.449 & 7522.64 & 9241.968 & 4794.36 & 8380.616 & 7934.026 & 8908.141 & 6486.096 & 6134.623 & 5656.99 & 6746.026 \\
\hline Modelo & 6467.424 & 5608.477 & 5649.271 & 7602.038 & 3517.418 & 4663.506 & 6013.287 & 6037.426 & 5492.71 & 3812.444 & 3694.912 & 3842.025 \\
\hline Pseudo $R^{2}$ Longford & $15.18 \%$ & $16.95 \%$ & $24.90 \%$ & $17.74 \%$ & $26.63 \%$ & $44.35 \%$ & $24.21 \%$ & $32.23 \%$ & $15.32 \%$ & $37.85 \%$ & $34.68 \%$ & $43.05 \%$ \\
\hline Deviance Nulo & 44455.213 & 73614.412 & 46969.55 & 51516.848 & 59513.127 & 30108.111 & 29337.834 & 63688.148 & 194049.373 & 46839.722 & 34039.971 & 34003.374 \\
\hline Deviance Modelo & 44253.566 & 72609.356 & 46777.875 & 51226.329 & 58671.615 & 29699.549 & 29153.494 & 62953.292 & 192450.612 & 46365.692 & 33664.55 & 33485.38 \\
\hline Diferencia & 201.647 & 1005.056 & 191.675 & 290.519 & 841.512 & 408.562 & 184.34 & 734.856 & 1598.761 & 474.03 & 375.421 & 517.994 \\
\hline
\end{tabular}

Nota 1: Coeficiente significativo al: *0.01**0.05***0.1

Nota 2: Los bajos coeficientes de ajuste del pseudo $\mathrm{R}^{2}$ de Longford (1993) para Finlandia, Canadá, Singapur y Corea se explica a partir del modelo no condicionado, el cual sugería evaluar características familiares y de alumnos para estos países con el fin de mejorar la explicación de la varianza no conocida. Fuente: Elaboración propia a partir de PISA 2012 (OCDE, 2014). 
Pese a la existencia de brechas en el logro académico entre niños y niñas no se logra establecer una tendencia que sugiera que las posiciones alcanzadas por los países están condicionadas por el sexo del alumno. Mientras que en Chile, Colombia, Brasil, Bélgica, Alemania, Francia, Shanghái y Uruguay los niños obtienen en promedio entre 4.32 y 15.41 puntos más que las niñas, en Finlandia, Singapur y Canadá el efecto diferencial está a favor de las niñas, y en Corea del Sur no prevalecen diferencias. Sin embargo, la diferencia de 14.69 puntos entre los niños y las niñas en Colombia evidencia una heterogeneidad en el aprendizaje que merece mayor atención, en el sentido en que mejorar en las pruebas implica que los niveles de aprendizaje deben ser uniformes.

La proporción de planteles (públicos o privados) en cada país logra reflejar parcialmente sus estructuras educativas. Así, los resultados econométricos no están condicionados por esta proporción debido a que, en países como Shanghái, Brasil, Colombia, Uruguay y Canadá, poco más del 64\% de sus instituciones son públicas pero el logro académico obtenido por sus alumnos es inferior al obtenido en las instituciones privados en aproximadamente 40 puntos, encontrando la mayor disimilitud en Brasil con 67.284 puntos y la menor en Colombia con 19.626. Además, la estructura educativa analizada muestra que cerca del $75 \%$ de las instituciones en Chile y Bélgica son privadas manteniendo en firme la brecha de resultados.

Los alumnos que han repetido al menos una vez algún curso del ISCED 2 o que se han ausentado de clases obtienen resultados ostensiblemente más bajos que aquellos que nunca han cometido el hecho. En Finlandia, Canadá y Singapur los alumnos que repiten curso obtienen entre 68.89, 65.17 y 61.16 puntos menos que los que nunca han repetido algún curso, mientras el absentismo se asocia con bajos rendimientos, en especial, en Corea del Sur donde el logro académico disminuye en promedio 74.22 para los alumnos que se han ausentado al menos una vez.

Lo anterior confirma el grave inconveniente que el absentismo y la repetición de curso escolar forja sobre los procesos formativos de los alumnos. Sin embargo, pese a que en todos los países las dos variables son significativas, exceptuando a Brasil para la variable absentismo escolar, y la diferencia entre logros académicos es considerablemente alta, no es posible interpretar que la eliminación de estos eventos conllevaría al aumento en el logro académico en la medida que no es un causante directo, sino más bien un efecto asociado al retraso cognitivo del alumno frente a los que siguen un proceso educativo continuo, y por tanto, se deben pensar en políticas diferenciadoras orientadas a prestar atención particular a los alumnos con estas características que convencionalmente se dan por cuestiones de vinculación temprana al mercado laboral o falta de recursos económicos en el hogar.

La localización del plantel en zonas distintas a las ciudades (incluyendo capitales) se asocia con diferenciales negativos. La puntuación obtenida por los alumnos de Chile, Corea y Uruguay que estudian en planteles ubicados en ciudades oscila entre 17.9 y 22.72 puntos más que aquellos pertenecientes a planteles ubicados en municipios, pueblos o villas, entendidos como zonas de menor desarrollo, pero sin poder precisar que son rurales debido a que las pruebas PISA no aportan esta información. En Colombia, 18.83 puntos es la diferencia promedio entre el logro académico de los alumnos que asisten a planteles ubicados en ciudades frente a los situados en municipios, pueblos o villas, convirtiéndose en una preocupación latente por cuanto el $21.10 \%$ de los alumnos evaluados en esta muestra están fuera de las ciudades. Los resultados introducen el componente de emplazamiento que bien merece ser profundizado en posteriores investigaciones dado que, al ser significativo en cuatro de los doce países ${ }^{2}$, el estudio sólo permite concluir que la calidad educativa no es homogénea

\footnotetext{
${ }^{2}$ El coeficiente igual a cero en Shanghái y Singapur es resultado de la no variabilidad de la muestra evaluada, puesto que el porcentaje de los alumnos ubicados en las ciudades es del $100 \%$.
} 
entre instituciones, soslayando las características propias de los planteles como por ejemplo la dotación escolar o la calidad de los maestros en zonas distantes a las urbes.

El nivel educacional del padre y la madre se relacionan positivamente con el logro académico del alumno. En países como Finlandia, Francia, Corea y Canadá el nivel de escolaridad de la madre tiene mayor magnitud que en el resto de países, lo cual sugiere que aquellos alumnos con madres tituladas de profesiones técnicas o universitarias obtienen 20.377, 9.407, 9.093 y 9.214 puntos más en comparación con los alumnos que tienen madres tituladas con educación secundaria o menos.

Por su parte, la escolaridad del padre tiene coeficientes igualmente positivos encontrando que los de mayor magnitud están en las economías presentadas previamente. Esta característica también muestra que la brecha de logro académico atribuida a la escolaridad de los padres se reduce a medida que el análisis pasa de países con mejores puntajes a menores, sugiriendo que la presencia de padres más cualificados en términos formativos, están en las economías con mayor desarrollo económico y, por tanto, el efecto es mayor cuando no se presenta la característica.

La posición ocupacional de los padres tiene dos alternativas de interpretación. La primera propone que padres/madres ocupados son un proxy del nivel económico del hogar y, por tanto, se espera que envíen a sus hijos al plantel, siendo el signo esperado positivo. La segunda alternativa permite explicar la presencia de signos negativos pues se relaciona con la asistencia académica provista en el hogar por parte de los padres. Hogares donde algunas de las figuras paternas tengan tiempo libre pueden emplear una parte en acompañar al alumno en la elaboración de actividades académicas y así ejercer una mayor atención al desarrollo cognitivo.

En Alemania y Uruguay el estado ocupacional de la madre no es significativo mientras para el resto sí, y al contar con signo positivo, la reflexión se esboza de acuerdo a la primera alternativa de interpretación de la variable, encontrando adicionalmente que gran parte de los países con madres ocupadas son aquellos que ocupan posiciones altas e intermedias de PISA y que, a su vez, cuentan con una mayor magnitud en comparación con los países de posiciones bajas. En relación a la ocupación del padre, el signo negativo se obtuvo en Chile, mientras en Bélgica, Brasil y Finlandia el signo es positivo.

Una variable adicional que permite consolidar el argumento sobre asistencia académica por parte de la madre y el padre hacia el alumno es la que enuncia si vive con ambas figuras. Los resultados apuntan al hecho de que en hogares donde está presente la característica los alumnos obtienen entre 4.856 (alumnos chilenos) y 18.109 (alumnos coreanos) puntos más que aquellos alumnos en convivencia monoparental o con otros miembros del hogar. En relación con los alumnos colombianos que conviven con ambas figuras familiares, obtienen en promedio 14.433 puntos más, siendo el cuarto país con mayor magnitud.

La variable dotación hogar condensa la tenencia por parte de los alumnos de al menos uno de ciertos elementos sindicados positivamente con el logro académico como escritorios, ordenadores, literatura clásica, internet y un lugar de estudio. En todos los países, exceptuando Corea, persiste la significancia estadística, donde la magnitud del coeficiente es mayor en Finlandia, Francia y Canadá con una diferencia positiva entre 28.296, 27.414 y 20.445 puntos a favor de los alumnos que poseen al menos uno de los elementos, mientras en Brasil, Colombia, Shanghái y Uruguay la magnitud está entre 4.232 y 7.230. No obstante, como limitante de la investigación está el hecho de no evaluar de manera particular cada uno de los elementos asociados con la variable, recogiendo así efectos conjuntos. 
El tamaño de la clase es la variable que más interés ha despertado entre los teóricos, pues goza de gran preeminencia en los estudios educativos pese a que sus disímiles resultados dista de unificar consensos. En Alemania (49.839), Francia (60.015), Brasil (29.314), Canadá (20.445), Finlandia (53.467), Uruguay (13.440) y Colombia (14.828) la diferencia está a favor de los cursos con más de 25 alumnos, mientras Chile, Corea, Singapur, Bélgica y Shanghái no existe diferencia.

Si bien, los resultados son contrarios a lo esperado, la generalidad presentada en los países sugiere conformar tamaños de clase mayor a 25 alumnos, pero la pregunta secular que no es contestada en la investigación debido a que la concentración de alumnos en determinados cursos no permite una mayor segmentación de los mismo es: ¿cuál es el tamaño óptimo de un curso de secundaria? Hoxby (2000) en su investigación plantea que la falta de consenso se explica por el grado de heterogeneidad de habilidades al interior de los cursos, práctica realizada habitualmente por los planteles en aras de diferenciar a los alumnos con alto y bajo potencial cognitivo.

El material de instrucción (posesión de textos académicos en el plantel) refleja el grado de acercamiento entre el alumno y el conocimiento al ser un instrumento de aprendizaje de fácil acceso, por tanto, su escasez debería reflejar una correspondencia negativa con el logro académico; resultado que se da con los alumnos de Francia, Bélgica y Colombia cuyo diferencial oscila entre 13.831 y 35.134. Por otra parte, no contar con mecanismos para actuar ante la escasez o falta de maestros cualificados para las competencias de matemáticas, ciencias y lenguaje por parte de los planteles está relacionado con logros académicos negativos sólo en Alemania, mientras para el resto de los países la variable no es significativa. La evidencia estadística sugiere que los alumnos alemanes que pertenecen a planteles que no están en capacidad de actuar ante la escasez de maestros obtienen 24.488 puntos menos.

En cuanto a las actividades extracurriculares, se relacionan positivamente con el logro académico. Realizar actividades que demanden concentración y agilidad mental como el juego de ajedrez o los clubes de matemáticas, informática y arte conllevan al desarrollo de habilidades cognitivas fuera de la clase que, si bien no es medible, refleja la capacidad de análisis y conexiones lógicas que los alumnos son capaces de efectuar en situaciones reales tal como lo presenta el informe de la OCDE (2014, p. 63,193,233) al explicar los niveles de competencia.

Con lo anterior, el fomento de estas actividades escolares en los planteles de Alemania, Canadá, Chile, Corea, Shanghái y Singapur guarda una relación positiva con el logro académico de sus alumnos, quienes obtienen entre 13.565 y 70.680 puntos más que los alumnos matriculados en planteles donde no se ofrece ninguna actividad; mientras en Brasil y Uruguay la provisión de actividades escolares se relacionan de forma negativa con el logro académico, resultados que para justificarlos requieren de un análisis profundo sobre la estructura educativa de estos países, no siendo el objetivo del documento pues no estamos en el orden de las humanidades y el ejercicio requerido para esto es cualitativo.

\subsection{Validación de los supuestos del modelo jerárquico.}

Los modelos jerárquicos sugieren el cumplimiento de tres supuestos iniciales que merecen ser examinados. El primero de los supuestos es la normalidad de la variable dependiente que justifica la agregación de los valores plausibles de las pruebas PISA en un solo término y para ello se emplea el estadístico conocido como coeficiente intercuantil, que de ser cercano a 1.3 se comprueba la hipótesis de normalidad en el conjunto de datos analizado.

$\mathrm{Al}$ examinar de manera independiente las puntuaciones promedio por competencias en cada país (tabla 3) se corrobora el supuesto de normalidad. Por tanto, pese a que las pruebas PISA evalúan 
las competencias en lectura, ciencias y matemáticas que a su vez están construidas con base a cinco valores plausibles, el proceso de agrupación a través de una media muestral que da como resultado un único valor correspondiente al logro académico promedio por alumno es acertado.

Tabla 3. Prueba de normalidad de puntuaciones promedio por competencias y países.

\begin{tabular}{c|ccccccccc}
\multicolumn{9}{c}{ Matemáticas } & \multicolumn{3}{c}{ Ciencias } & \multicolumn{2}{c}{ Lectura } \\
\hline Países & Intervalo & $\sigma$ & Coeficiente & Intervalo & $\sigma$ & Coeficiente & Intervalo & $\sigma$ & Coeficiente \\
Bélgica & 127.434 & 91.847 & $\mathbf{1 . 3 8 7}$ & 116.748 & 87.850 & $\mathbf{1 . 3 2 9}$ & 118.352 & 87.858 & $\mathbf{1 . 3 4 7}$ \\
Brasil & 106.870 & 78.079 & $\mathbf{1 . 3 6 9}$ & 101.362 & 74.217 & $\mathbf{1 . 3 6 6}$ & 106.980 & 77.744 & $\mathbf{1 . 3 7 6}$ \\
Canadá & 115.283 & 82.290 & $\mathbf{1 . 4 0 1}$ & 114.603 & 84.304 & $\mathbf{1 . 3 5 9}$ & 114.037 & 84.251 & $\mathbf{1 . 3 5 4}$ \\
Chile & 120.696 & 81.384 & $\mathbf{1 . 4 8 3}$ & 114.370 & 77.875 & $\mathbf{1 . 4 6 9}$ & 97.938 & 71.644 & $\mathbf{1 . 3 6 7}$ \\
Colombia & 95.108 & 71.901 & $\mathbf{1 . 3 2 3}$ & 96.419 & 70.749 & $\mathbf{1 . 3 6 3}$ & 102.537 & 74.340 & $\mathbf{1 . 3 7 9}$ \\
Alemania & 124.708 & 88.845 & $\mathbf{1 . 4 0 4}$ & 114.556 & 84.673 & $\mathbf{1 . 3 5 3}$ & 113.248 & 80.989 & $\mathbf{1 . 3 9 8}$ \\
Finlandia & 112.167 & 82.521 & $\mathbf{1 . 3 5 9}$ & 122.156 & 90.098 & $\mathbf{1 . 3 5 6}$ & 121.703 & 90.245 & $\mathbf{1 . 3 4 9}$ \\
Francia & 122.215 & 86.879 & $\mathbf{1 . 4 0 7}$ & 113.484 & 85.867 & $\mathbf{1 . 3 2 2}$ & 118.954 & 92.435 & $\mathbf{1 . 2 8 7}$ \\
Corea & 131.874 & 93.571 & $\mathbf{1 . 4 0 9}$ & 102.014 & 76.406 & $\mathbf{1 . 3 3 5}$ & 105.983 & 80.348 & $\mathbf{1 . 3 1 9}$ \\
Shanghái & 130.472 & 95.090 & $\mathbf{1 . 3 7 2}$ & 104.066 & 75.290 & $\mathbf{1 . 3 8 2}$ & 100.560 & 73.778 & $\mathbf{1 . 3 6 3}$ \\
Singapur & 144.493 & 100.085 & $\mathbf{1 . 4 4 4}$ & 143.417 & 100.086 & $\mathbf{1 . 4 3 3}$ & 130.637 & 94.264 & $\mathbf{1 . 3 8 6}$ \\
Uruguay & 115.361 & 82.929 & $\mathbf{1 . 3 9 1}$ & 116.188 & 86.251 & $\mathbf{1 . 3 4 7}$ & 115.320 & 85.899 & $\mathbf{1 . 3 4 2}$
\end{tabular}

Fuente: Elaboración propia a partir de PISA 2012 (OCDE, 2014).

El segundo supuesto hace referencia a que los componentes aleatorios y los valores pronosticados son ortogonales (independencia). Con las gráficas presentadas en el anexo 3 se comprueba la no existencia de tendencia y a partir del cálculo del coeficiente de correlación se afirma esta apreciación. En Bélgica, Colombia, Canadá y Finlandia en el nivel plantel se determinó una correlación promedia de 0.166 que en términos estadísticos no implica correlaciones altas y, por tanto, se puede concluir que también cumplen con el supuesto.

Finalmente, el tercer supuesto hace referencia a la normalidad del término error, contraste que se efectúa con un análisis gráfico de los residuales y es complementado con el test Jarque-Bera presentado en el anexo 4. Se encontró que la distribución de los errores es normal en Alemania, Chile, Brasil, Uruguay y Francia, mientras en Colombia, Canadá, Corea del Sur, Finlandia, China-Shanghái, Singapur y Bélgica el supuesto no se cumple para el nivel alumno y en el último país tampoco se cumple en el nivel plantel. El incumplimiento del supuesto está dado por la alta heterogeneidad en los logros académicos de los alumnos y su concentración en cada uno de los niveles de competencia; sin embargo, lo anterior no afecta la insesgadez de los coeficientes estimados.

\section{Conclusiones.}

Las características del alumno y del entorno familiar resaltan el papel del hogar como orientador del aprendizaje, pues convivir con la figura paterna y materna, el nivel de escolaridad de los padres y su vinculación laboral sugieren una relación positiva con el desempeño escolar. Por otra parte, es evidente la brecha en la calidad de la educación existente entre instituciones públicas y privadas aun cuando la proporción al interior de ciertos países evidencia estructuras educativas públicas.

La persistencia de habilidades homogéneas al interior de los cursos puede ser una característica controlada por los sistemas educativos de cada país, que al igual que el tamaño de los cursos se asocian positivamente con el logro académico. Si bien, una estrategia sería la conformación de cursos de acuerdo al nivel cognitivo del alumno, señalizados por sus resultados en pruebas nacionales y evaluaciones internas de los planteles educativos, las estrategias pedagógicas de acompañamiento escolar entre alumnos con habilidades disímiles se constituyen en una alternativa de política. 
Las características del entorno escolar vía dotación de elementos como la tenencia o no de textos, edificios y aulas de clase, muestra una asociación diferencial entre países y consistencia analítica pues su escasez se relaciona con una disminución del logro académico en países como Francia, Bélgica y Colombia. Así mismo, se encontró un hecho interesante que relaciona el tamaño de la clase y la escasez de aulas de clase. En países donde el tamaño de la clase es mayor y que a su vez se asocian con un logro académico positivo, la escasez de aulas se convierte en un factor que permite mantener esta relación. No obstante, la discusión sobre el tamaño de la clase sigue abierta por cuanto no se llega a un consenso dada la proporcionalidad de alumnos por cursos entre países, convirtiéndose en una extensión para futuros trabajos el profundizar sobre esta característica que se sugiere sea controlada desde la heterogeneidad de habilidades.

La investigación evalúa los efectos que la pedagogía y el compromiso educativo generan sobre el logro académico, encontrando una relación positiva entre las variables definidas para someter a prueba la hipótesis planteada. Simultáneamente, se encontró consistencia entre la intimidación escolar y las expectativas que los alumnos forman de los maestros, relación negativa que se da cuando las expectativas son bajas de los alumnos residentes en Corea, Singapur, Colombia, Alemania, Bélgica, Canadá, Chile y Uruguay. Estas características sugieren que en los planteles donde existe una percepción de intimidación escolar y una baja relación entre actores educativos se impide el aprendizaje, se dan bajos resultados en las pruebas.

Propender a una disminución del absentismo y la repitencia escolar; mejorar las condiciones estructurales de la economía para que el acceso a la educación y la inserción al mercado laboral sean procesos dinámicos, en especial para los padres de familia; fomentar practicas académicas extracurriculares; crear cátedras morales y éticas que estimulen el respeto y la tolerancia hacia los compañeros con el fin de disminuir la intimidación escolar; solidificar el vínculo maestro-alumno a partir de ejercicios de comunicación fuera de los espacios académicos son factores que permiten sugerir un perfil educativo meta. Adicionalmente, debe reconocerse socialmente la importancia que los maestros tienen en la educación y, por tanto, es decisivo brindar condiciones que permita elevar sus titulaciones académicas.

Los modelos multinivel son una herramienta metodológica que contribuye en la correcta evaluación de las características asociadas al proceso formativo de los alumnos dada su capacidad para dividir la varianza desconocida en niveles, siendo dos los analizados en la investigación, los alumnos y los planteles. Así mismo, se encontró una consistencia en las estimaciones en relación a la preponderancia de profundizar en las características que estuvieran asociados a un determinado nivel y que tuvieran un mayor predominio en la explicación de la varianza en cada país.

Los resultados disímiles entre países producto del análisis econométrico permiten corroborar la hipótesis planteada. Es evidente que las variables explicativas examinadas se relacionan de distintas formas al interior de cada contexto educativo y en ciertos casos guardan cierto grado de homogeneidad en cuanto a su relación con la variable dependiente.

El mejoramiento de la calidad de educación es un proceso arduo, que conlleva a repensar en los modelos educativos que se siguen al interior de cada país y para ello es importante tener en cuenta fundamentos teóricos y empíricos que permitan a los hacedores de política enfocarse en las características del entorno familiar o escolar que se asocien a una mejora en el logro académico del alumnado. Con lo anterior, a nivel técnico se recomienda el uso de los modelos jerárquicos para analizar el fenómeno de la educación, tanto en niveles como en métodos más sofisticados como los modelos jerárquicos probabilísticos, pues métodos convencionales no captan las unidades de análisis de manera adecuada. 
Finalmente, los especialistas en educación fueron los primeros en usar estructuras jerárquicas en sus análisis, por tanto, es claro que la multidisciplinariedad de los campos se reforzaría con experimentos económicos que permitiesen acercar a los investigadores sociales a respuestas tentativas que la sola base de datos PISA de logro académico no permiten abarcar, generando el siguiente interrogante: ¿ existe un nivel adicional que mejore la comprensión del logro académico?

\section{Agradecimientos}

Esta investigación es un subproducto derivado del proyecto: "Determinantes del Logro Académico en los Alumnos de Secundaria: Un Análisis de la Incidencia del Entorno Escolar a través de las Pruebas Pisa 2012 con Modelos Lineales Jerárquicos”, financiado por el Instituto Colombiano para el Fomento de la Educación Superior (ICFES). Las ideas, opiniones, tesis y argumentos expresados son de autoría exclusiva del autor y no representa el punto de vista del Instituto.

\section{Referencias}

Barrientos, M.J. (2008). Calidad de la educación pública y logro académico en Medellín 2004-2006. Una aproximación por regresión intercuartil. Lecturas de Economía, 68, 121-144.

Becker, G.S. (1962). Irrational behavior and economic theory. Journal of Political Economy, 70(1), $1-13$.

Betts, J.R., \& Shkolnik, J.L. (2000). The effects of ability grouping on student achievement and resource allocation in secondary schools. Economics of Education Review, 19(1), 1-15.

Bryk, A.S., \& Raudenbush, S.W. (1992). Hierarchical linear models for social and behavioral research: Applications and data analysis methods. Newbury Park, CA: SAGE.

Calero, J., \& Escardíbul, J.O. (2007). Evaluación de servicios educativos: el rendimiento en los centros públicos y privados medido en PISA-2003. IEB Working Paper 2007/07.

Cano, F. (2006). Factores de logro cognitivo en la escuela primaria colombiana. Estudio realizado sobre una muestra de planteles grados $3^{\circ}, 5^{\circ}$ (1993-1994). Estudios Sobre Eficacia Escolar En Iberoamérica, 15, 33-60.

Casas, A.F., Gamboa, L.F., \& Piñeros, L.J. (2002). El efecto escuela en Colombia, 1999-2000. Bogotá, Colombia: Editorial Universidad del Rosario.

Cervini, R. (2004). Nivel y variación de la equidad en la educación media de Argentina. Revista Iberoamericana de Educación, 34(1), 1-18.

Cohen, D., Raudenbush, S., \& Ball, D. (2003). Resources, instruction, and research. Educational Evaluation and Policy Analysis, 25(2), 119-142.

Coleman, J. (1968). The concept of equality of educational opportunity. Harvard Educational Review, 38(1), 7-22. 
Cordero, J. M., Manchón, C., \& Simancas, R. (2012). Análisis de los condicionantes del rendimiento educativo de los alumnos españoles en PISA 2009 mediante técnicas multinivel. Presupuesto y Gasto Público, 67, 71-96.

Correa, J. J. (2004). Determinantes del rendimiento educativo de los estudiantes de secundaria en Cali: un análisis multinivel. Sociedad y Economía, 6, 81-105.

Denison, E. F. (1962). Sources of economic growth in the United States and the alternatives before us. New York, USA: Committee for Economic Development.

Ermisch, J., \& Francesconi, M. (2001). Family matters: Impacts of family background on educational attainments. Economica, 68(270), 137-156.

Galor, O., \& Zeira, J. (1993). Income distribution and macroeconomics. The Review of Economic Studies, 60(1), 35-52.

Gaviria, A., \& Barrientos, J. H. (2001). Determinantes de la calidad de la educación en Colombia. Archivos de Economía, 1(159), 88.

Gaviria, J. L., \& Castro, M. (2005). Modelos jerárquicos lineales. Serie Cuadernos de Estadística, (29).

Ghouali, H. (2007). El acompañamiento escolar y educativo en Francia. Revista Mexicana de Investigación Educativa, 12(32), 207-242.

Goldstein, H. (1995). Multilevel Statistical Models 2nd edition. Bristol, United Kingdom: Hodder Education.

Hanushek, E. (1971). Teacher characteristics and gains in student achievement: Estimation using micro data. The American Economic Review, 61(2), 280-288.

Hanushek, E. A. (2008). The Economic benefits of improved teacher quality. In Governance and performance of education systems (pp. 107-135). Springer.

Hanushek, E., Markman, J., \& Rivkin, S.G. (2003). Does peer ability affect student achievement? Journal of Applied Econometrics, 18(5), 527-544.

Hernández, F., Rosário, P., de Tejada, J.D., Martínez, P., \& Ruiz, E. (2006). Promoción del aprendizaje estratégico y competencias de aprendizaje en estudiantes de primero de universidad: evaluación de una intervención. Revista de Investigación Educativa, 24(2), 615632.

Hox, J. (2002). Quantitative methodology series. Multilevel Analysis Techniques and Applications. Mahwah, NJ, US: Lawrence Erlbaum Associates Publishers.

Hoxby, C.M. (2000). The effects of class size on student achievement: New evidence from population variation. The Quarterly Journal of Economics, 115(4), 1239-1285. 
Jencks, C. (1972). Inequality: A reassessment of the effect of family and schooling in America. New York, USA: Basic Books.

Kane, T.J. (2006). Chapter 23 Public Intervention in Post-Secondary Education. Handbook of the Economics of Education, 2, 1369-1401.

Lazear, E.P. (1980). Family Background and Optimal Schooling Decision. National Bureau of Economic Research Cambridge, Mass., USA.

Lee, J.W., \& Barro, R.J. (2001). Schooling quality in a cross-section of countries. Economica, 68(272), 465-488.

López, S.F. (2010). El papel de los incentivos y los docentes en la calidad de la educación oficial de Medellín. Trabajo de Grado No Publicado de Maestría (Meritoria), Universidad de Antioquia, Medellín, Colombia.

Martín, E.L., Asencio, E.N., Ordóñez, X.G., \& Romero, S.J. (2009). Estudio de variables determinantes de eficiencia a través de los modelos jerárquicos lineales en la evaluación PISA 2006: el caso de España. Education Policy Analysis Archives, 17(1), 1-24.

Mayer, S.E., \& Peterson, P.E. (1999). Earning and learning: How schools matter. Brookings Institution Press.

Núñez, J., Steiner, R., Cadena, X., \& Pardo, R. (2002). ¿Cuáles colegios ofrecen mejor educación en Colombia? Archivos de Economía, 193, 1-56.

Organisation for Economic Co-operation and Development (2014). PISA 2012 results: What students know and can do (volume I, revised edition, February 2014): Student performance in mathematics, reading and science. OECD Publishing.

Orrego, M. (2009). Incidencia del entorno escolar en el rendimiento académico de los alumnos de secundaria: comparaciones internacionales con base en las pruebas de PISA 2006. Universidad del Valle. Cali: Facultad de Ciencias Sociales y Económicas. Economía.

Piñeros, L. J., \& Rodríguez, A. (1998). Los insumos escolares en la educación secundaria y su efecto sobre el rendimiento académico de los estudiantes: un estudio en Colombia. Washington, DC: The World Bank/Latin America and the Caribbean Region/Department of Human Development.

Ramos, R., Duque, J.C., \& Nieto, S. (2012). Decomposing the rural-urban differential in student achievement in Colombia using PISA microdata. Working Paper 23, 1-28.

Raudenbush, S.W., \& Bryk, A.S. (2002). Hierarchical linear models: Applications and data analysis methods (Vol. 1). Sage.

Sarmiento, A., Becerra, L., \& González, J. I. (2000). La incidencia del plantel en el logro educativo del alumno y su relación con el nivel socioeconómico. Coyuntura Social, Fedesarrollo, 264, 53-63. 
Schultz, T.W. (1961). Investment in human capital. The American Economic Review, 51(1), 1-17.

Snijders, T., \& Bosker, R. (1999). Multilevel modeling: An introduction to basic and advanced multilevel modeling. London, United Kingdom: SAGE.

Spady, W.G. (1973). The Impact of School Resources on Students. Review of Research in Education, 1(1), 135-177.

Tobón, D., Posada, H. M., \& Ríos, P. (2009). Determinants of the performance of the schools in Medellín in the High-School Graduation-Year Test (ICFES). Cuadernos de Administración, 22(38), 311-333.

Vivas, H. (2008). Educación, background familiar y calidad de los entornos locales en Colombia (Tesis Doctoral). Departamento d’Economia Aplicada, Universitat Autónoma de Barcelona (UAB), Barcelona.

Zambrano, J. C. (2013). Análisis multinivel del rendimiento escolar en matemáticas para cuarto grado de Educación Básica Primaria en Colombia. Sociedad y Economía, 25, 205-235. 


\section{Anexos}

Anexo 1. Variables seleccionadas y metodología de construcción.

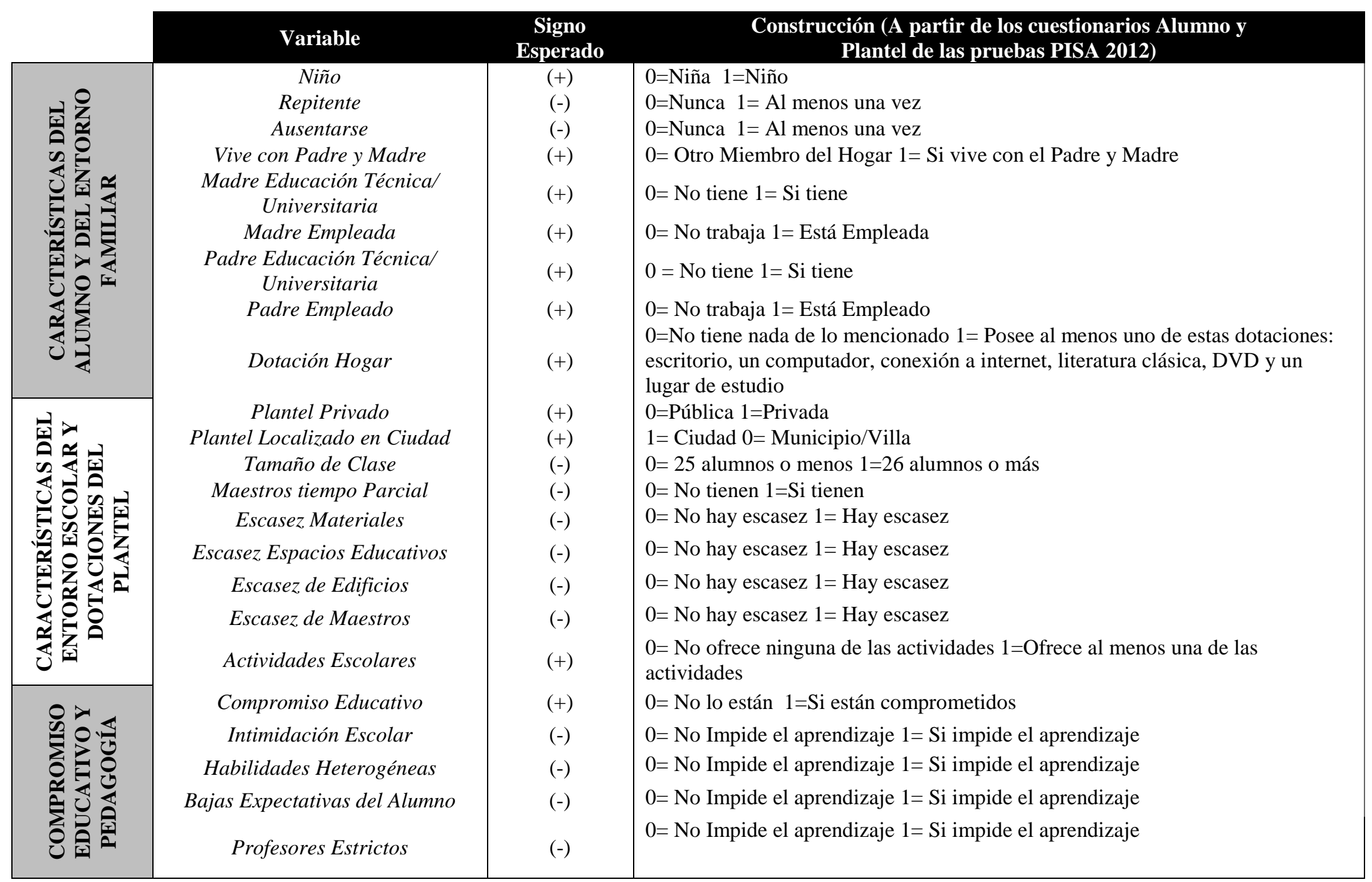

Fuente: Elaboración propia con base en los Codebook de PISA 2012 (OCDE, 2014). 
Anexo 2. Estadísticos descriptivos con muestra agregada.

\begin{tabular}{|c|c|c|c|c|c|}
\hline $\begin{array}{c}\text { VARIABLES } \\
\text { Variable Dependiente }\end{array}$ & Mínimo & Máximo & Medía & Desv. Típica & $\begin{array}{c}\text { Número de } \\
\text { Observaciones }\end{array}$ \\
\hline Logro Académico & 176.669 & 859.766 & 511.996 & 97.268 & 62769 \\
\hline \multicolumn{6}{|l|}{ Regresores Nivel Alumno } \\
\hline Niño & 0 & 1 & 0.4874699 & 0.4998470 & 62769 \\
\hline Repitente & 0 & 1 & 0.0786535 & 0.2691993 & 62769 \\
\hline Ausentarse & 0 & 1 & 0.1120298 & 0.3154057 & 62769 \\
\hline Vive Con Padre Y Madre & 0 & 1 & 0.8029601 & 0.3977659 & 62769 \\
\hline Madre Con Educación Técnica o Universitaria & 0 & 1 & 0.6006627 & 0.4897661 & 62769 \\
\hline Madre Empleada & 0 & 1 & 0.9536077 & 0.2103349 & 62769 \\
\hline Padre Con Educación Técnica o Universitaria & 0 & 1 & 0.6326849 & 0.4820772 & 62769 \\
\hline Padre Empleado & 0 & 1 & 0.9695550 & 0.1718096 & 62769 \\
\hline Dotación Hogar & 0 & 1 & 0.3653077 & 0.4815202 & 62769 \\
\hline \multicolumn{6}{|l|}{ Regresores Nivel Plantel } \\
\hline Plantel Privado & 0 & 1 & 0.2539311 & 0.4352621 & 62769 \\
\hline Plantel Localizado En Ciudad & 0 & 1 & 0.5470853 & 0.4977820 & 62769 \\
\hline Tamaño De Clase & 0 & 1 & 0.6371776 & 0.4808180 & 62769 \\
\hline Maestros Tiempo Parcial & 0 & 1 & 0.8659848 & 0.3406714 & 62769 \\
\hline Escasez Materiales & 0 & 1 & 0.4800937 & 0.4996076 & 62769 \\
\hline Escasez de Maestros & 0 & 1 & 0.3340662 & 0.4716667 & 62769 \\
\hline Actividades Escolares & 0 & 1 & 0.1010053 & 0.3013381 & 62769 \\
\hline Compromiso Educativo & 0 & 1 & 0.2793576 & 0.4486871 & 62769 \\
\hline Intimidación Escolar & 0 & 1 & 0.7581927 & 0.4281815 & 62769 \\
\hline Habilidades Heterogéneas & 0 & 1 & 0.8845131 & 0.3196112 & 62769 \\
\hline Bajas Expectativas Del Alumno & 0 & 1 & 0.5677165 & 0.4953972 & 62769 \\
\hline Profesores Estrictos & 0 & 1 & 0.6902611 & 0.4623896 & 62769 \\
\hline
\end{tabular}

Fuente: Elaboración propia a partir de PISA 2012 (OCDE, 2014). 
Anexo 3. Supuesto de ortogonalidad residuales.

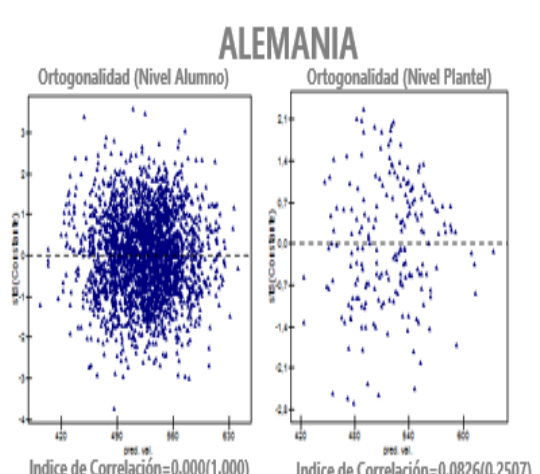
BRASIL

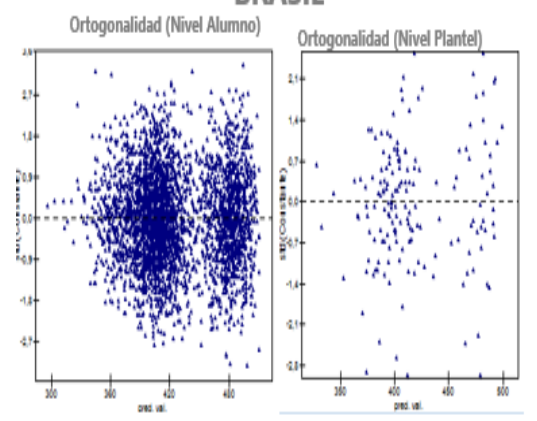

Indice de Correlación $=0.000(1.000) \quad$ Indice de Correlación $=0.0891(0.2539$ FRANCIA
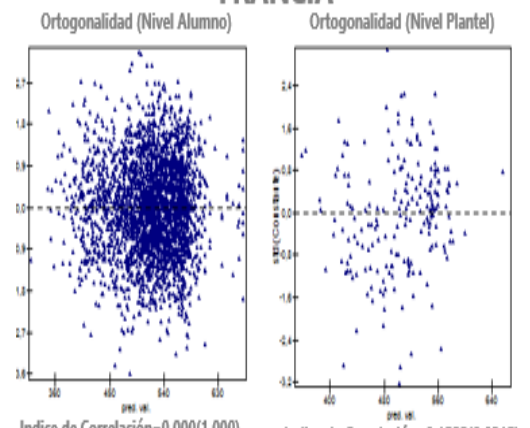

Indice de Corralación $=0.152200315$ )

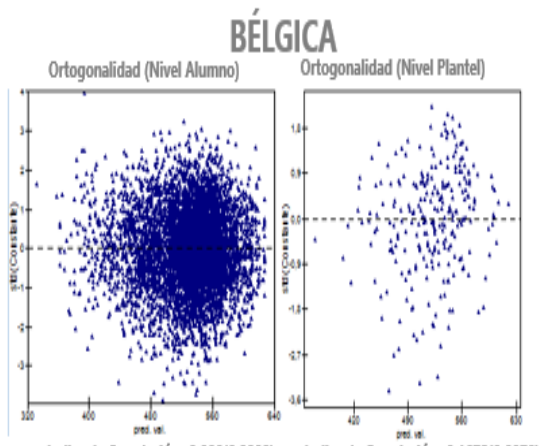

Indice de Correlacón =0.000(0.9999) Indice de
CANADÁ
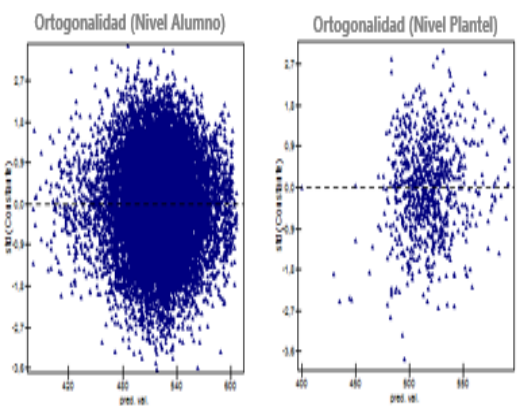

Indice de Correläín=0.000(1.000) Indice de Corre
CHINA-SHANGHAI

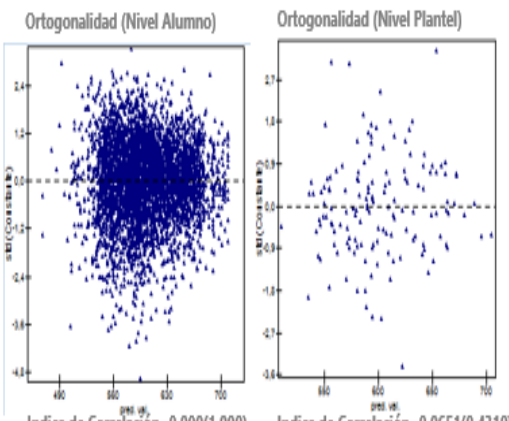

atiede Fuente: Elaboración propia a partir de PISA 2012 (OCDE, 2014).
CHILE
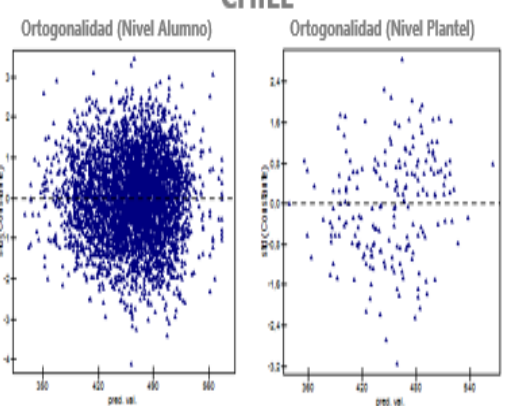

Indice de Correlacín $=0.16390 .0224$

COREA DEL SUR

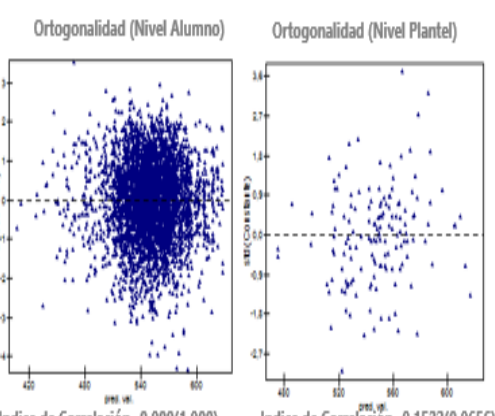

SINGAPUR

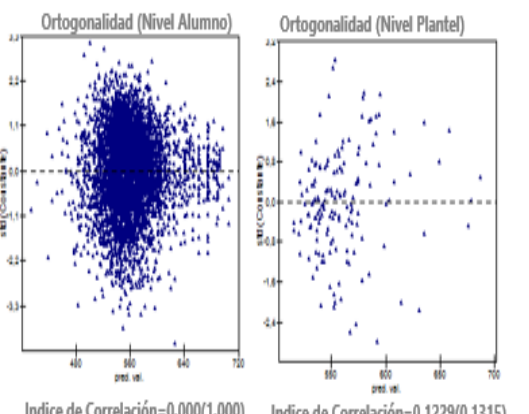

Indice de Correlación $=0,000(1,000)$ Indice de Correlación $=0,01229(0,1315)$
COLOMBIA

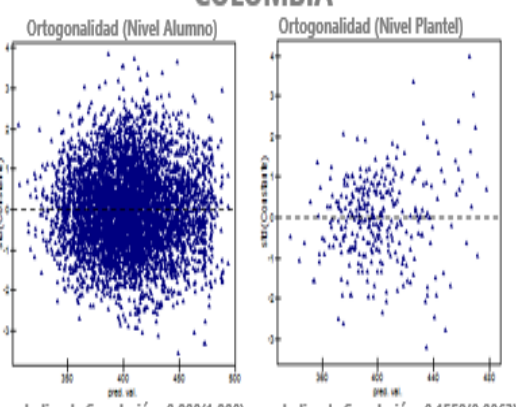

FINLANDIA

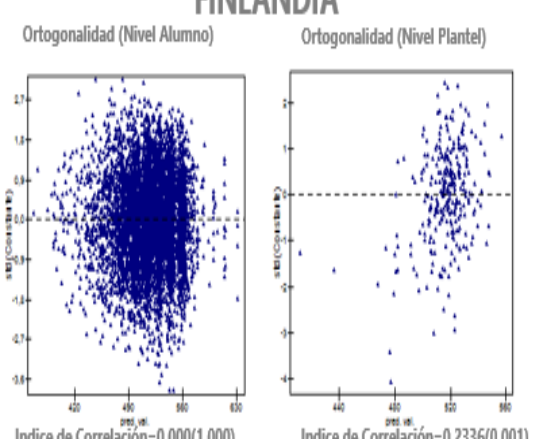

URUGUAY

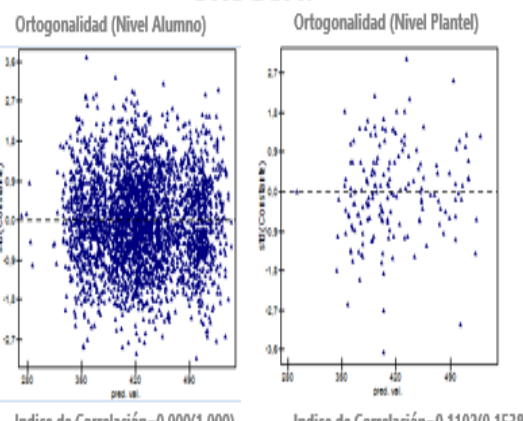


Anexo 4. Supuesto de normalidad residuales.

ALEMANIA

Probabilidad Normal (Nivel Alumnol Probabilidad Normal (Nivel Plantel)

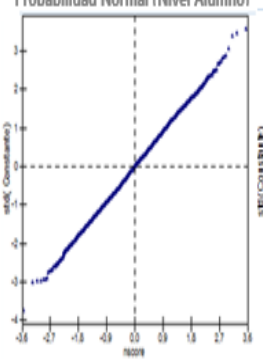

Jarque-Bera $=0.592512(0.743597)$ BRASIL

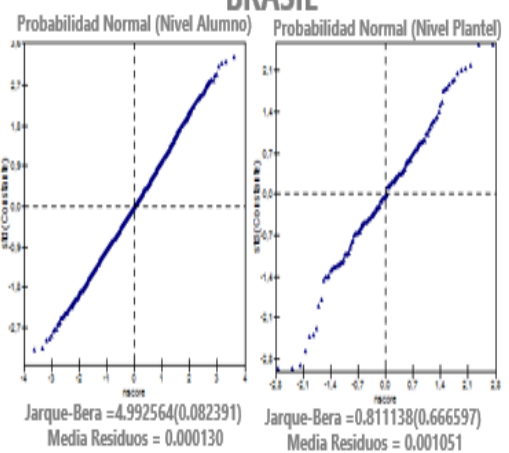
FRANCIA

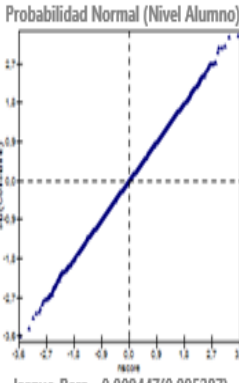

Jarque-Bera $=0.009447(0.995287$ Media Residuos $=-0.000141$
BÉELGICA

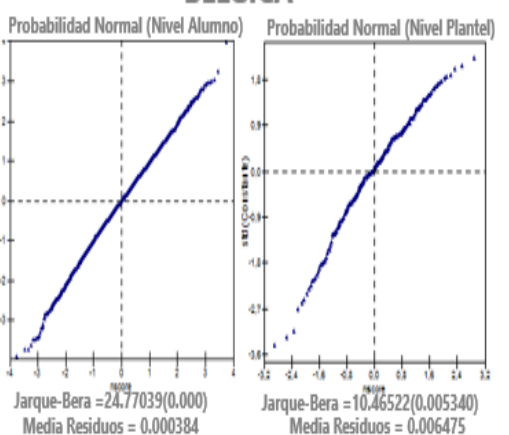

CANADÁ

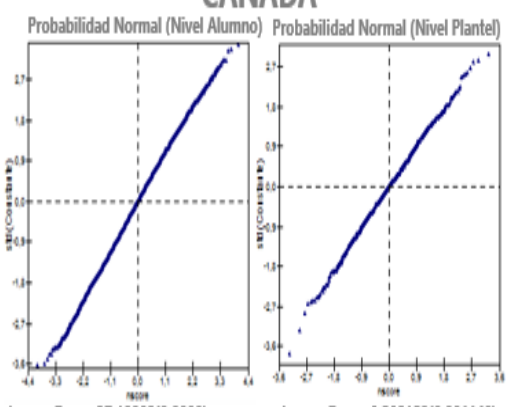

Jarque-Bera $=37,10008(0.0000)$

Jarque-Bera $=0.208189(0.901140)$
Media Residuos $=0.001049$ CHINA-SHANGHÁI

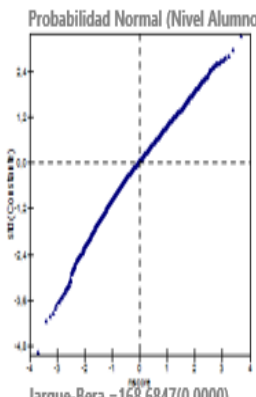

Jarque-Bera $=168.6847(0,0000$

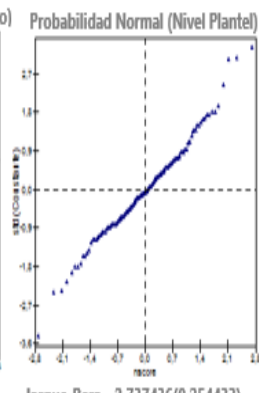

arque Bera $=2.7373436(0.254433)$
CHILE

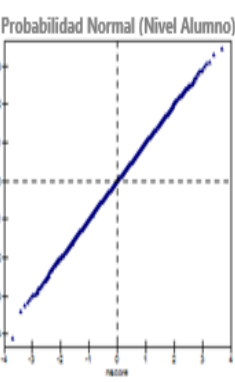

Jarque-Bera $=0.978113(0.613205)$
Media Residuos $=2.65-05$ COREA DEL SUR

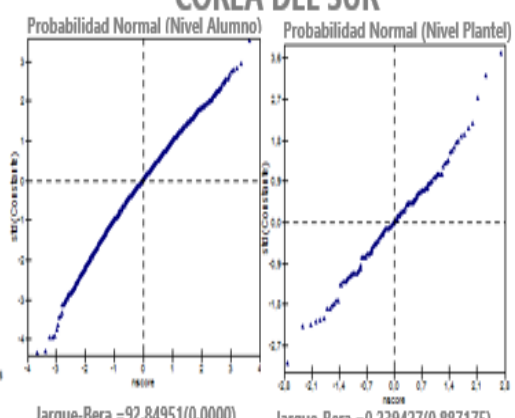

arque-Bera $=92.84951(0.0000)$

Jarque-Bera $=0.239427(0.887175)$ SINGAPUR

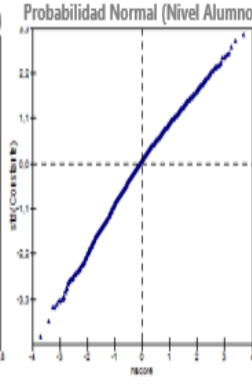

Jarque-Bera $=117.1706(0.0000)$
Media Residuos $=4-67 \mathrm{e}-05$

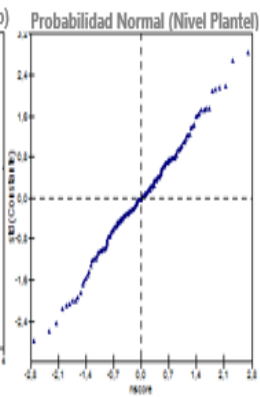
Media Residuos $=0.00050$

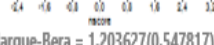

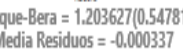

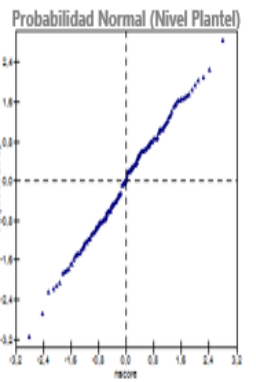

in

(1)

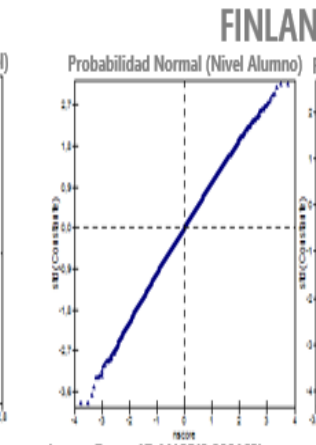

Jarque Bera = 17.4.41855(0.0000163)

$$
\text { URUGUAY }
$$
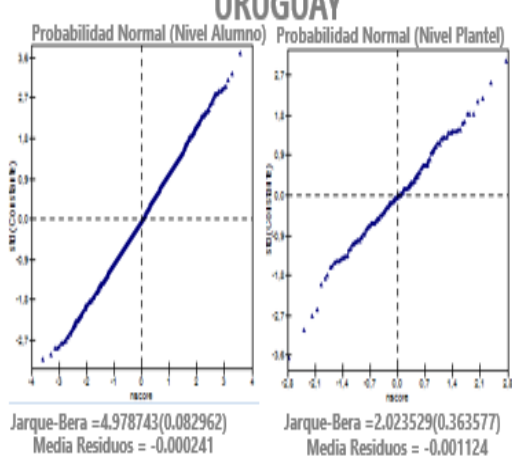

Fuente: Elaboración propia a partir de PISA 2012 (OCDE, 2014). 